\title{
Geometric Analysis of the Some Rectified Archimedean Solids Spaces via Their Isometry Groups
}

\author{
Temel Ermiş*
}

\begin{abstract}
There are two main motivations in this article. First, we give the new metrics and the metric spaces whose unit spheres are Rectified Archimedean Solids. Then, using the general technique which is quite simple, we show that isometry group of $\mathbb{R}^{3}$ endowed with these new metrics are the semi direct product of the translation group $T(3)$ of $\mathbb{R}^{3}$ with the Euclidean symmetry groups of Rectified Archimedean Solids.

Keywords: Polyhedrons; Metric geometry; Isometry group; Octahedral group; Icosahedral group; Rectified Archimedean solids. AMS Subject Classification (2020): Primary: 51B20; 51N25; Secondary: 51F99; 51K05; 51K99.

*Corresponding author
\end{abstract}

\section{Introduction}

The main idea of the geometric group theory is to treat finitely-generated groups as geometric objects. It is understandable efforts to bring together geometry and algebra. Thus, one can easily analyze the mathematical system thanks to transformations on this system. The transformation preserve designated features of the geometric structure. The set of transformations compose the groups consisting of the symmetries of geometric objects. The symmetry group for a physical object (or polyhedron) is the set of ways that object can be repositioned so that it maintains its symmetry, or looks the same. Any object of any dimension has such a group of symmetries. The set of three-dimensional objects called polyhedrons possess many beautiful symmetries and accordingly form interesting symmetry groups under their rotations. So, the excellent symmetry of the polyhedrons (or solids) have made them perfect models for the studying on symmetries. A polyhedron is called regular if all its faces are equal and regular polygons. It is called semi-regular if all its faces are regular polygons and all its vertices are equal. An irregular polyhedron is defined by polygons that are composed of elements that are not all equal. A regular polyhedron is called Platonic solid, a semi-regular polyhedron is called Archimedean solid and an irregular polyhedron is called Catalan solid. Archimedean solids are generally derived from Platonic solids by truncating. In geometry, any polyhedron is associated with a second dual figure, where the vertices of one correspond to the faces of the other and the edges between pairs of vertices of one correspond to the edges between pairs of faces of the other. According to this information, a Catalan solid, or Archimedean dual, is a dual polyhedron to an Archimedean solid. Similarly, rectified Archimedean solids been created by applying the rectify operation to one of the Archimedean solids.

Polyhedrons have been studied by scientists and artists during many years, because their symmetries are very interesting $[8,9]$. The Platonic Solids have the greatest geometric symmetry of any shapes in existence. If one would like compare the insights of today's particle physics with any earlier philosophy, it could be only the philosophy of Plato, since the particles of today's physics are representations of symmetry groups - that is what quantum theory teaches us - and hence the particles resemble the regular Platonic polyhedra [15]. Nowadays, modern technology and multi-disciplinary studies have confirmed that many nanoparticles take on some Platonic solids and other convex symmetric polyhedrons. [1], [4], [11], [14]. The classification including physical and chemical properties of the elements in the nature is made by means of the periodic table well-known. The classification which

Received : 07-11-2019, Accepted : 07-10-2020 
includes physical properties of elementary particles in the sub-atomic world is not made of monotype table or geometrical patterns. Untill 1960' s, The question "Is there classification for elementary particles in the sub-atomic world such as the periodic table in chemistry?" was investigated. There is not the monotype classification which includes the physical properties (electric charge, mass, spin, etc. ) of elementary particles, but it is known that different configurations so-called "eightfold way [1], [11]" determines geometrical patterns of elementary particles . Therefore, convex symmetric polyhedrons and their metric structures which will be studied in the next section of this article could provide new applications in various studies of the supermultiples based on the physical properties of elementary particles [11]. In the light of the above, convex symmetric polyhedrons have been observed not only in physical and geometric group theory studies, but also investigations on medicine, astronomy, architecture, etc... For examples, Many viruses, such as the HIV, have the shape of a regular icosahedron (see Figure 1).

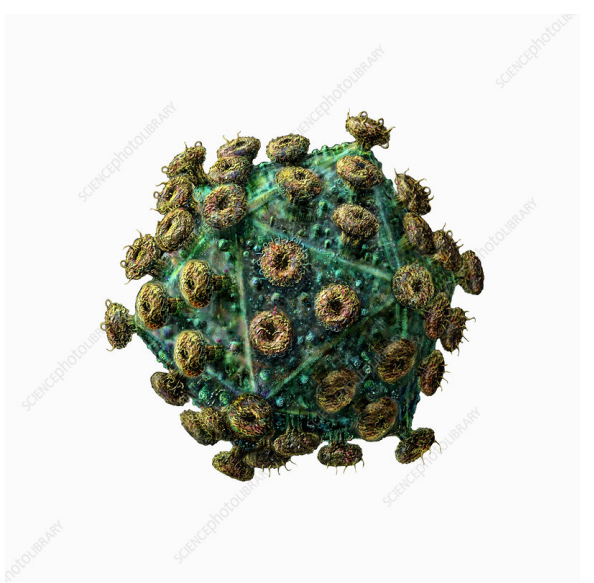

Figure 1: AIDS virus particle protein shell

https://www.sciencephoto.com/media/248680/view

In the following section, we give the new metrics which unit spheres of $\mathbb{R}^{3}$ furnishing by these metrics are rectified truncated cube, rectified truncated octahedron, rectified truncated dodecahedron and rectified truncated icosahedron in the three dimensional analytic space. We know that the set of solutions

$$
P=\left\{\begin{array}{cccc} 
& a_{11} x_{1}+\cdots+a_{1 n} x_{n} & \leq & b_{1} \\
\left(x_{1}, x_{2}, \ldots, x_{n}\right) \in \mathbb{R}^{n}: & \vdots & \vdots & \vdots \\
& a_{m 1} x_{1}+\cdots+a_{m n} x_{n} & \leq & b_{m}
\end{array}\right\}
$$

to a system

$$
\begin{array}{ccc}
a_{11} x_{1}+\cdots+a_{1 n} x_{n} & \leq & b_{1} \\
\vdots & \vdots & \vdots \\
a_{m 1} x_{1}+\cdots+a_{m n} x_{n} & \leq & b_{m}
\end{array}
$$

of finitely many lineer inequalities (here $a_{i j}$ and $b_{i}$ are real numbers for $i=1, \ldots, n$ and $j=1, \ldots, m$ ) is called a polyhedron. Furthermore, each the equation $a_{j i} x_{i}=b_{j}$ can be considered as the surface plane equations of the polyhedron. So, the surface plane equations of the polyhedron must be determined to find the metric for any polyhedron. However, to do this, the following steps are important:

Step 1 : The polyhedrons are placed to have the most appropriate symmetry in the coordinate system. Thus, the corner points of the polyhedrons will be symmetrically coordinated.

Step 2 : Using the symmetrical corner points, surface plane equations of polyhedrons are found, which equations of planes will be symmetrical.

Thanks to these two steps, the distance function for the polyedron will provide the axiom of symmetry in the metric space.

Step 3 : Finally, it should be given general relation including equations of the planes containing faces of polyhedrons. To ensure the non-negative axiom in the metric space, this general relation must be given with the aid of the absolute value function.

Consequently, the given general relation will automatically provide the triangular inequality, thanks to these three steps and because the polyhedron is convex and symmetrical. 
In functional analysis, for a convex symmetric polyhedron given in $\mathbb{R}^{n}$, it is known that the existence of the norm whose unit sphere is this convex symmetric polyhedron [17]. But, we do not know how this norm is defined. In the next section, we give the new metrics in cartesian form which unit spheres of the spaces endowing with these metrics are Rectified Truncated Cube, Rectified Truncated Octahedron, Rectified Truncated Dodecahedron and Rectified Truncated Icosahedron, respectively.

\section{Some Rectified Archimedean Solids}

Rectified Archimedean Solids been created by applying the rectify operation to one of the Archimedean solids. Rectification is a form of truncation where each vertex is truncated up to the midpoints of the edges meeting at that vertex. This operation is typically applied to one of the Platonic solids, but it can also be applied to a non-regular polyhedron as long as the midpoints of the edges meeting at each vertex are coplanar. If the non-regular polyhedron is convex, then the resulting polyhedron will not intersect itself.

\subsection{Rectified Truncated Cube}

The rectified truncated cube is a polyhedron. It has 38 faces: 8 equilateral triangles, 24 isosceles triangles, and 6 octagons. Topologically, the triangles corresponding to the cube's vertices are always equilateral, although the octagons, while having equal edge lengths, do not have the same edge lengths with the equilateral triangles, having different but alternating angles, causing the other triangles to be isosceles instead.
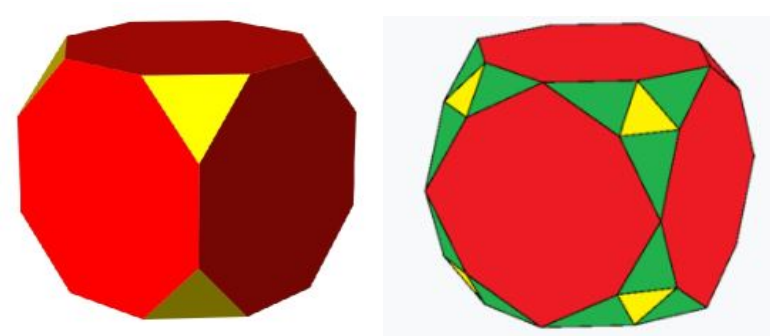

Figure 2: Truncated Cube and Rectified Truncated Cube

Corner Points of Rectified Truncated Cube: We will denote the corner points of Rectified Truncated Cube with $v_{i}=(x, y, z)$ for $i=0, \ldots, 35$. These corner points can be coordinated as in the following table for $c=\sqrt{2} / 2$;

\begin{tabular}{r|r|r|r|r|r|r|r|r|r|r|r|} 
& \multicolumn{1}{|c|}{$x$} & \multicolumn{1}{|c|}{$z$} & $z$ & & $x$ & $y$ & $z$ & & $x$ & $y$ & $z$ \\
\hline$v_{0}$ & 1 & 0 & 1 & $v_{12}$ & $c$ & $c$ & 1 & $v_{24}$ & -1 & $c$ & $c$ \\
$v_{1}$ & 1 & 0 & -1 & $v_{13}$ & $c$ & $c$ & 1 & $v_{25}$ & -1 & $c$ & $-c$ \\
$v_{2}$ & -1 & 0 & 1 & $v_{14}$ & $c$ & $-c$ & 1 & $v_{26}$ & -1 & $-c$ & $c$ \\
$v_{3}$ & -1 & 0 & -1 & $v_{15}$ & $c$ & $-c$ & 1 & $v_{27}$ & -1 & $-c$ & $-c$ \\
$v_{4}$ & 1 & 1 & 0 & $v_{16}$ & $-c$ & $c$ & 1 & $v_{28}$ & $c$ & 1 & $c$ \\
$v_{5}$ & 1 & -1 & 0 & $v_{17}$ & $-c$ & $c$ & 1 & $v_{29}$ & $c$ & 1 & $-c$ \\
$v_{6}$ & -1 & 1 & 0 & $v_{18}$ & $-c$ & $-c$ & 1 & $v_{30}$ & $c$ & -1 & $c$ \\
$v_{7}$ & -1 & -1 & 0 & $v_{19}$ & $-c$ & $-c$ & 1 & $v_{31}$ & $c$ & -1 & $-c$ \\
$v_{8}$ & 0 & 1 & 1 & $v_{20}$ & 1 & $c$ & $c$ & $v_{32}$ & $-c$ & 1 & $c$ \\
$v_{9}$ & 0 & 1 & -1 & $v_{21}$ & 1 & $c$ & $-c$ & $v_{33}$ & $-c$ & 1 & $-c$ \\
$v_{10}$ & 0 & -1 & 1 & $v_{22}$ & 1 & $-c$ & $c$ & $v_{34}$ & $-c$ & -1 & $c$ \\
$v_{11}$ & 0 & -1 & -1 & $v_{23}$ & 1 & $-c$ & $-c$ & $v_{35}$ & $-c$ & -1 & $-c$
\end{tabular}

Surface plane equations passing the corner points given above : It is known that the equation of a plane with nonzero normal vector $\vec{N}=(A, B, C)$ in $\mathbb{R}^{3}$ is $A x+B y+C z+D=0$. However, in order to obtain a unit sphere, $D=-1$ will be taken. We show that the normal vectors of the plane's equations passing through the specified corner points in the following table; let be $\lambda_{0}=-1+\sqrt{2}, \lambda_{1}=1 / 2$; 


\begin{tabular}{|c|c|c|c|c|c|c|c|}
\hline Corner Points & $A$ & $B$ & $C$ & Corner Points & $A$ & $B$ & $C$ \\
\hline$v_{12}, v_{20}, v_{28}$ & $\lambda_{0}$ & $\lambda_{0}$ & $\lambda_{0}$ & $v_{0}, v_{14}, v_{22}$ & $\lambda_{1}$ & $-\lambda_{0} \lambda_{1}$ & $\lambda_{1}$ \\
\hline$v_{13}, v_{29}, v_{21}$ & $\lambda_{0}$ & $\lambda_{0}$ & $-\lambda_{0}$ & $v_{0}, v_{20}, v_{12}$ & $\lambda_{1}$ & $\lambda_{0} \lambda_{1}$ & $\lambda_{1}$ \\
\hline$v_{14}, v_{30}, v_{22}$ & $\lambda_{0}$ & $-\lambda_{0}$ & $\lambda_{0}$ & $v_{1}, v_{13}, v_{21}$ & $\lambda_{1}$ & $\lambda_{0} \lambda_{1}$ & $-\lambda_{1}$ \\
\hline$v_{15}, v_{23}, v_{31}$ & $\lambda_{0}$ & $-\lambda_{0}$ & $-\lambda_{0}$ & $v_{1}, v_{23}, v_{15}$ & $\lambda_{1}$ & $-\lambda_{0} \lambda_{1}$ & $-\lambda_{1}$ \\
\hline$v_{16}, v_{32}, v_{24}$ & $-\lambda_{0}$ & $\lambda_{0}$ & $\lambda_{0}$ & $v_{2}, v_{16}, v_{24}$ & $-\lambda_{1}$ & $\lambda_{0} \lambda_{1}$ & $\lambda_{1}$ \\
\hline$v_{17}, v_{25}, v_{33}$ & $-\lambda_{0}$ & $\lambda_{0}$ & $-\lambda_{0}$ & $v_{2}, v_{26}, v_{18}$ & $-\lambda_{1}$ & $-\lambda_{0} \lambda_{1}$ & $\lambda_{1}$ \\
\hline$v_{18}, v_{26}, v_{34}$ & $-\lambda_{0}$ & $-\lambda_{0}$ & $\lambda_{0}$ & $v_{3}, v_{19}, v_{27}$ & $-\lambda_{1}$ & $-\lambda_{0} \lambda_{1}$ & $-\lambda_{1}$ \\
\hline$v_{19}, v_{35}, v_{27}$ & $-\lambda_{0}$ & $-\lambda_{0}$ & $-\lambda_{0}$ & $v_{3}, v_{25}, v_{17}$ & $-\lambda_{1}$ & $\lambda_{0} \lambda_{1}$ & $-\lambda_{1}$ \\
\hline$v_{4}, v_{21}, v_{29}$ & $\lambda_{1}$ & $\lambda_{1}$ & $-\lambda_{0} \lambda_{1}$ & $v_{8}, v_{12}, v_{28}$ & $\lambda_{0} \lambda_{1}$ & $\lambda_{1}$ & $\lambda_{1}$ \\
\hline$v_{4}, v_{28}, v_{20}$ & $\lambda_{1}$ & $\lambda_{1}$ & $\lambda_{0} \lambda_{1}$ & $v_{8}, v_{32}, v_{16}$ & $-\lambda_{0} \lambda_{1}$ & $\lambda_{1}$ & $\lambda_{1}$ \\
\hline$v_{5}, v_{22}, v_{30}$ & $\lambda_{1}$ & $-\lambda_{1}$ & $\lambda_{0} \lambda_{1}$ & $v_{9}, v_{17}, v_{33}$ & $-\lambda_{0} \lambda_{1}$ & $\lambda_{1}$ & $-\lambda_{1}$ \\
\hline$v_{5}, v_{31}, v_{23}$ & $\lambda_{1}$ & $-\lambda_{1}$ & $-\lambda_{0} \lambda_{1}$ & $v_{9}, v_{29}, v_{13}$ & $\lambda_{0} \lambda_{1}$ & $\lambda_{1}$ & $-\lambda_{1}$ \\
\hline$v_{6}, v_{24}, v_{32}$ & $-\lambda_{1}$ & $\lambda_{1}$ & $\lambda_{0} \lambda_{1}$ & $v_{10}, v_{18}, v_{34}$ & $-\lambda_{0} \lambda_{1}$ & $-\lambda_{1}$ & $\lambda_{1}$ \\
\hline$v_{6}, v_{33}, v_{25}$ & $-\lambda_{1}$ & $\lambda_{1}$ & $-\lambda_{0} \lambda_{1}$ & $v_{10}, v_{30}, v_{14}$ & $\lambda_{0} \lambda_{1}$ & $-\lambda_{1}$ & $\lambda_{1}$ \\
\hline$v_{7}, v_{27}, v_{35}$ & $-\lambda_{1}$ & $-\lambda_{1}$ & $-\lambda_{0} \lambda_{1}$ & $v_{11}, v_{15}, v_{31}$ & $\lambda_{0} \lambda_{1}$ & $-\lambda_{1}$ & $-\lambda_{1}$ \\
\hline$v_{7}, v_{34}, v_{26}$ & $-\lambda_{1}$ & $-\lambda_{1}$ & $\lambda_{0} \lambda_{1}$ & $v_{11}, v_{35}, v_{19}$ & $-\lambda_{0} \lambda_{1}$ & $-\lambda_{1}$ & $-\lambda_{1}$ \\
\hline$v_{0}, v_{12}, v_{8}, v_{16}, v_{2}, v_{18}, v_{10}, v_{14}$ & 0 & 0 & 1 & $v_{0}, v_{22}, v_{5}, v_{23}, v_{20}, v_{1}, v_{21}, v_{4}$ & 1 & 0 & 0 \\
\hline$v_{1}, v_{15}, v_{11}, v_{19}, v_{3}, v_{17}, v_{9}, v_{13}$ & 0 & 0 & -1 & $v_{2}, v_{24}, v_{6}, v_{25}, v_{3}, v_{27}, v_{7}, v_{26}$ & -1 & 0 & 0 \\
\hline$v_{4}, v_{29}, v_{9}, v_{33}, v_{6}, v_{32}, v_{8}, v_{28}$ & 0 & 1 & 0 & $v_{5}, v_{30}, v_{10}, v_{34}, v_{7}, v_{35}, v_{11}, v_{31}$ & 0 & -1 & 0 \\
\hline
\end{tabular}

Surface plane equations of Rectified Truncated Cube, which normal vectors given in the above tables, can be generalized as follows;

$$
\begin{aligned}
& \lambda_{0}(|x|+|y|+|z|)=1, \quad \lambda_{1}\left(|x|+\lambda_{0}|y|+|z|\right)=1, \quad|x|=1, \quad|y|=1 \\
& \lambda_{1}\left(\lambda_{0}|x|+|y|+|z|\right)=1, \quad \lambda_{1}\left(|x|+|y|+\lambda_{0}|z|\right)=1, \quad|z|=1
\end{aligned}
$$

To bound the polyhedron to these planes, the maximum of these general relations (generalized plane equations) must be taken. Thus, we define the distance function which the unit sphere of analytical space furnishing by this metric is Rectified Truncated Cube containing all these surface plane equations. This distance function will be denoted by $d_{\text {RTC }}$.

Definition 2.1. Let $X=\left(x_{1}, y_{1}, z_{1}\right)$ and $Y=\left(x_{2}, y_{2}, z_{2}\right)$ be any two points in the $\mathbb{R}^{3}$, for brevity, $\mathbf{X}_{12}=\left|x_{1}-x_{2}\right|$, $\mathbf{Y}_{12}=\left|y_{1}-y_{2}\right|$ and $\mathbf{Z}_{12}=\left|z_{1}-z_{2}\right|$. Then $d_{\mathbf{R T C}}: \mathbb{R}^{3} \times \mathbb{R}^{3} \rightarrow[0, \infty)$ is defined by

$$
d_{\mathbf{R T C}}(X, Y)=\max \left\{F_{i}: i=1, \ldots, 7\right\}
$$

where

$$
\begin{aligned}
& F_{1}=\lambda_{1}\left(\lambda_{0} \mathbf{X}_{12}+\mathbf{Y}_{12}+\mathbf{Z}_{12}\right), \quad F_{3}=\lambda_{1}\left(\mathbf{X}_{12}+\mathbf{Y}_{12}+\lambda_{0} \mathbf{Z}_{12}\right), \quad F_{5}=\mathbf{X}_{12}, \quad F_{6}=\mathbf{Y}_{12} \\
& F_{2}=\lambda_{1}\left(\mathbf{X}_{12}+\lambda_{0} \mathbf{Y}_{12}+\mathbf{Z}_{12}\right), \quad F_{4}=\lambda_{0}\left(\mathbf{X}_{12}+\mathbf{Y}_{12}+\mathbf{Z}_{12}\right), \quad F_{7}=\mathbf{Z}_{12}
\end{aligned}
$$

such that $\lambda_{0}=-1+\sqrt{2}, \lambda_{1}=1 / 2$.

\subsection{Rectified Truncated Octahedron}

The rectified truncated octahedron is a polyhedron. It has 38 faces: 24 isosceles triangles, 6 squares, and 8 hexagons. Topologically, the squares corresponding to the octahedron's vertices are always regular, although the hexagons, while having equal edge lengths, do not have the same edge lengths with the squares, having different but alternating angles, causing the triangles to be isosceles instead.

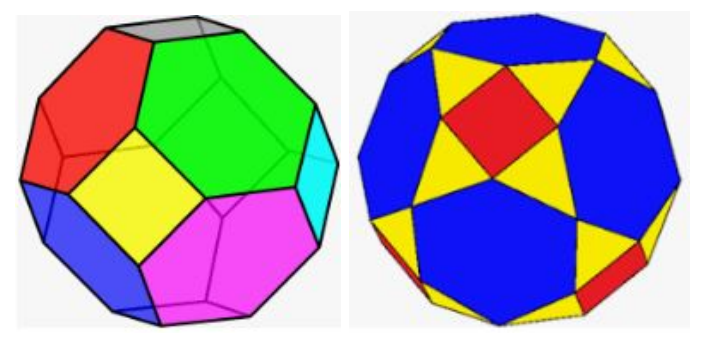

Figure 3: Truncated Octahedron and Rectified Truncated Octahedron 
Corner Points of Rectified Truncated Octahedron: We will denote the corner points of Rectified Truncated Octahedron with $v_{i}=(x, y, z)$ for $i=0, \ldots, 35$. These corner points can be coordinated as in the following table for $c_{0}=1 / 4$ and $c_{1}=3 / 4$;

\begin{tabular}{r|r|r|r|r|r|r|r|r|r|r|r|} 
& \multicolumn{1}{|c|}{$x$} & \multicolumn{1}{|c|}{$z$} & \multicolumn{1}{|c|}{$z$} & & $x$ & $y$ & $z$ & & $x$ & $y$ & \multicolumn{1}{c}{$z$} \\
\hline$v_{0}$ & $c_{0}$ & $c_{0}$ & 1 & $v_{12}$ & -1 & $c_{0}$ & $c_{0}$ & $v_{24}$ & $c_{1}$ & 0 & $c_{1}$ \\
$v_{1}$ & $c_{0}$ & $c_{0}$ & -1 & $v_{13}$ & -1 & $c_{0}$ & $-c_{0}$ & $v_{25}$ & $c_{1}$ & 0 & $-c_{1}$ \\
$v_{2}$ & $c_{0}$ & $-c_{0}$ & 1 & $v_{14}$ & -1 & $-c_{0}$ & $c_{0}$ & $v_{26}$ & $-c_{1}$ & 0 & $c_{1}$ \\
$v_{3}$ & $c_{0}$ & $-c_{0}$ & -1 & $v_{15}$ & -1 & $-c_{0}$ & $-c_{0}$ & $v_{27}$ & $-c_{1}$ & 0 & $-c_{1}$ \\
$v_{4}$ & $-c_{0}$ & $c_{0}$ & 1 & $v_{16}$ & $c_{0}$ & 1 & $c_{0}$ & $v_{28}$ & $c_{1}$ & $c_{1}$ & 0 \\
$v_{5}$ & $-c_{0}$ & $c_{0}$ & -1 & $v_{17}$ & $c_{0}$ & 1 & $-c_{0}$ & $v_{29}$ & $c_{1}$ & $-c_{1}$ & 0 \\
$v_{6}$ & $-c_{0}$ & $-c_{0}$ & 1 & $v_{18}$ & $c_{0}$ & -1 & $c_{0}$ & $v_{30}$ & $-c_{1}$ & $c_{1}$ & 0 \\
$v_{7}$ & $-c_{0}$ & $-c_{0}$ & -1 & $v_{19}$ & $c_{0}$ & -1 & $-c_{0}$ & $v_{31}$ & $-c_{1}$ & $-c_{1}$ & 0 \\
$v_{8}$ & 1 & $c_{0}$ & $c_{0}$ & $v_{20}$ & $-c_{0}$ & 1 & $c_{0}$ & $v_{32}$ & 0 & $c_{1}$ & $c_{1}$ \\
$v_{9}$ & 1 & $c_{0}$ & $-c_{0}$ & $v_{21}$ & $-c_{0}$ & 1 & $-c_{0}$ & $v_{33}$ & 0 & $c_{1}$ & $-c_{1}$ \\
$v_{10}$ & 1 & $-c_{0}$ & $c_{0}$ & $v_{22}$ & $-c_{0}$ & -1 & $c_{0}$ & $v_{34}$ & 0 & $-c_{1}$ & $c_{1}$ \\
$v_{11}$ & 1 & $-c_{0}$ & $-c_{0}$ & $v_{23}$ & $-c_{0}$ & -1 & $-c_{0}$ & $v_{35}$ & 0 & $-c_{1}$ & $-c_{1}$
\end{tabular}

The normal vectors $\vec{N}=(A, B, C)$ of surface plane equations passing the corner points given above: Let be $\lambda_{0}=2 / 3, \lambda_{1}=4 / 9$ and $\lambda_{2}=8 / 9$;

\begin{tabular}{|c|c|c|c|c|c|c|c|}
\hline Corner Points & $A$ & $B$ & $C$ & Corner Points & $A$ & $B$ & $C$ \\
\hline$v_{0}, v_{2}, v_{24}$ & $\lambda_{1}$ & 0 & $\lambda_{2}$ & $v_{12}, v_{14}, v_{26}$ & $-\lambda_{2}$ & 0 & $\lambda_{1}$ \\
\hline$v_{0}, v_{32}, v_{4}$ & 0 & $\lambda_{1}$ & $\lambda_{2}$ & $v_{12}, v_{30}, v_{13}$ & $-\lambda_{2}$ & $\lambda_{1}$ & 0 \\
\hline$v_{1}, v_{5}, v_{33}$ & 0 & $\lambda_{1}$ & $-\lambda_{2}$ & $v_{13}, v_{27}, v_{15}$ & $-\lambda_{2}$ & 0 & $-\lambda_{1}$ \\
\hline$v_{1}, v_{25}, v_{3}$ & $\lambda_{1}$ & 0 & $-\lambda_{2}$ & $v_{14}, v_{15}, v_{31}$ & $-\lambda_{2}$ & $-\lambda_{1}$ & 0 \\
\hline$v_{2}, v_{6}, v_{34}$ & 0 & $-\lambda_{1}$ & $\lambda_{2}$ & $v_{16}, v_{20}, v_{32}$ & 0 & $\lambda_{2}$ & $\overline{\lambda_{1}}$ \\
\hline$v_{3}, v_{35}, v_{7}$ & 0 & $-\lambda_{1}$ & $-\lambda_{2}$ & $v_{16}, v_{28}, v_{17}$ & $\lambda_{1}$ & $\lambda_{2}$ & 0 \\
\hline$v_{4}, v_{46}, v_{6}$ & $-\lambda_{1}$ & 0 & $\lambda_{2}$ & $v_{17}, v_{33}, v_{21}$ & 0 & $\lambda_{2}$ & $-\lambda_{1}$ \\
\hline$v_{5}, v_{7}, v_{27}$ & $-\lambda_{1}$ & 0 & $-\lambda_{2}$ & $v_{18}, v_{19}, v_{29}$ & $\lambda_{1}$ & $-\lambda_{2}$ & 0 \\
\hline$v_{8}, v_{9}, v_{28}$ & $\lambda_{2}$ & $\lambda_{1}$ & 0 & $v_{18}, v_{34}, v_{22}$ & 0 & $-\lambda_{2}$ & $\lambda_{1}$ \\
\hline$v_{8}, v_{24}, v_{10}$ & $\lambda_{2}$ & 0 & $\lambda_{1}$ & $v_{19}, v_{23}, v_{35}$ & 0 & $-\lambda_{2}$ & $-\lambda_{1}$ \\
\hline$v_{9}, v_{11}, v_{25}$ & $\lambda_{2}$ & 0 & $-\lambda_{1}$ & $v_{20}, v_{21}, v_{30}$ & $-\lambda_{1}$ & $\lambda_{2}$ & 0 \\
\hline$v_{10}, v_{29}, v_{11}$ & $\lambda_{2}$ & $-\lambda_{1}$ & 0 & $v_{22}, v_{31}, v_{23}$ & $-\lambda_{1}$ & $-\lambda_{2}$ & 0 \\
\hline$v_{0}, v_{4}, v_{6}, v_{2}$ & 0 & 0 & 1 & $v_{12}, v_{13}, v_{15}, v_{14}$ & -1 & 0 & 0 \\
\hline$v_{1}, v_{3}, v_{7}, v_{5}$ & 0 & 0 & -1 & $v_{16}, v_{17}, v_{21}, v_{20}$ & 0 & 1 & 0 \\
\hline$v_{8}, v_{10}, v_{11}, v_{9}$ & 1 & 0 & 0 & $v_{18}, v_{22}, v_{23}, v_{19}$ & 0 & -1 & 0 \\
\hline$v_{0}, v_{24}, v_{8}, v_{28}, v_{16}, v_{32}$ & $\lambda_{0}$ & $\lambda_{0}$ & $\lambda_{0}$ & $v_{4}, v_{32}, v_{20}, v_{30}, v_{12}, v_{26}$ & $-\lambda_{0}$ & $\lambda_{0}$ & $\lambda_{0}$ \\
\hline$v_{1}, v_{33}, v_{17}, v_{28}, v_{9}, v_{25}$ & $\lambda_{0}$ & $\lambda_{0}$ & $-\lambda_{0}$ & $v_{5}, v_{27}, v_{13}, v_{30}, v_{21}, v_{33}$ & $-\lambda_{0}$ & $\lambda_{0}$ & $-\lambda_{0}$ \\
\hline$v_{2}, v_{34}, v_{18}, v_{29}, v_{10}, v_{24}$ & $\lambda_{0}$ & $-\lambda_{0}$ & $\lambda_{0}$ & $v_{6}, v_{26}, v_{14}, v_{31}, v_{22}, v_{34}$ & $-\lambda_{0}$ & $-\lambda_{0}$ & $\lambda_{0}$ \\
\hline$v_{3}, v_{25}, v_{11}, v_{29}, v_{19}, v_{35}$ & $\lambda_{0}$ & $-\lambda_{0}$ & $-\lambda_{0}$ & $v_{7}, v_{35}, v_{23}, v_{31}, v_{15}, v_{27}$ & $-\lambda_{0}$ & $-\lambda_{0}$ & $-\lambda_{0}$ \\
\hline
\end{tabular}

Surface plane equations of Rectified Truncated Octahedron, which normal vectors given in the above tables, can be generalized as follows;

$$
\begin{array}{lll}
\lambda_{1}|x|+\lambda_{2}|y|=1, & \lambda_{1}|y|+\lambda_{2}|x|=1, & \lambda_{1}|z|+\lambda_{2}|x|=1 \\
\lambda_{1}|x|+\lambda_{2}|z|=1, & \lambda_{1}|y|+\lambda_{2}|z|=1, & \lambda_{1}|z|+\lambda_{2}|y|=1 \\
\lambda_{0}(|x|+|y|+|z|)=1, & |x|=1,|y|=1, \quad|z|=1, &
\end{array}
$$

Thus, we define the distance function which the unit sphere of analytical space furnishing by this metric is Rectified Truncated Octahedron containing all these surface plane equations. This distance function will be denoted by $d_{\mathbf{R T O}}$.

Definition 2.2. Let $X=\left(x_{1}, y_{1}, z_{1}\right)$ and $Y=\left(x_{2}, y_{2}, z_{2}\right)$ be any two points in the $\mathbb{R}^{3}$, for brevity, $\mathbf{X}_{12}=\left|x_{1}-x_{2}\right|$, $\mathbf{Y}_{12}=\left|y_{1}-y_{2}\right|$ and $\mathbf{Z}_{12}=\left|z_{1}-z_{2}\right|$. Then $d_{\mathbf{R T O}}: \mathbb{R}^{3} \times \mathbb{R}^{3} \rightarrow[0, \infty)$ is defined by

$$
d_{\mathbf{R T O}}(X, Y)=\max \left\{F_{i}: i=1, \ldots, 10\right\}
$$


where

$$
\begin{array}{lll}
F_{1}=\lambda_{1} \mathbf{X}_{12}+\lambda_{2} \mathbf{Y}_{12} & F_{3}=\lambda_{1} \mathbf{Y}_{12}+\lambda_{2} \mathbf{X}_{12} & F_{5}=\lambda_{1} \mathbf{Z}_{12}+\lambda_{2} \mathbf{X}_{12} \\
F_{2}=\lambda_{1} \mathbf{X}_{12}+\lambda_{2} \mathbf{Z}_{12} & F_{4}=\lambda_{1} \mathbf{Y}_{12}+\lambda_{2} \mathbf{Z}_{12} & F_{6}=\lambda_{1} \mathbf{Z}_{12}+\lambda_{2} \mathbf{Y}_{12} \\
F_{7}=\lambda_{0}\left(\mathbf{X}_{12}+\mathbf{Y}_{12}+\mathbf{Z}_{12}\right) & F_{8}=\mathbf{X}_{12}, \quad F_{9}=\mathbf{Y}_{12}, & F_{10}=\mathbf{Z}_{12}
\end{array}
$$

such that $\lambda_{0}=2 / 3, \lambda_{1}=4 / 9$ and $\lambda_{2}=8 / 9$.

\subsection{Rectified Truncated Dodecahedron}

The rectified truncated dodecahedron is a polyhedron. It has 92 faces: 20 equilateral triangles, 60 isosceles triangles, and 12 decagons. Topologically, the triangles corresponding to the dodecahedrons's vertices are always equilateral, although the decagons, while having equal edge lengths, do not have the same edge lengths with the equilateral triangles, having different but alternating angles, causing the other triangles to be isosceles instead.
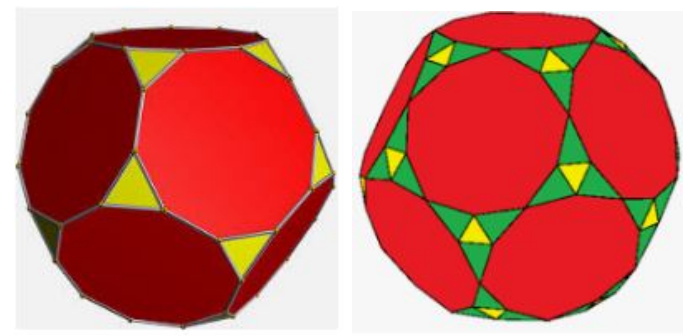

Figure 4: Truncated Dodecahedron and Rectified Truncated Dodecahedron

Corner Points of Rectified Truncated Dodecahedron: We will denote the corner points of Rectified Truncated Dodecahedron with $v_{i}=(x, y, z)$ for $i=0, \ldots, 89$. These corner points can be coordinated as in the following table for $c_{0}=\frac{-1+\sqrt{5}}{4}, c_{1}=\frac{\sqrt{5}}{5}, c_{2}=\frac{1}{2}, c_{3}=\frac{5+3 \sqrt{5}}{20}, c_{4}=\frac{15-\sqrt{5}}{20}, c_{5}=\frac{1+\sqrt{5}}{4}, c_{6}=\frac{2 \sqrt{5}}{5}, c_{7}=\frac{5+2 \sqrt{5}}{10}, c_{8}=1$ and $c_{9}=\frac{-5+3 \sqrt{5}}{20}$

\begin{tabular}{r|r|r|r|r|r|r|r|r|r|r|r|} 
& \multicolumn{1}{|c|}{$x$} & \multicolumn{1}{|c|}{$y$} & \multicolumn{1}{|c|}{$z$} & & $x$ & $y$ & \multicolumn{1}{|c}{$z$} & & $x$ & \multicolumn{1}{c}{$y$} & \multicolumn{1}{c}{$z$} \\
\hline$v_{0}$ & 0 & 0 & $c_{8}$ & $v_{15}$ & $c_{7}$ & $c_{9}$ & $-c_{0}$ & $v_{30}$ & 0 & $c_{1}$ & $c_{6}$ \\
$v_{1}$ & 0 & 0 & $-c_{8}$ & $v_{16}$ & $c_{7}$ & $-c_{9}$ & $c_{0}$ & $v_{31}$ & 0 & $c_{1}$ & $-c_{6}$ \\
$v_{2}$ & $c_{8}$ & 0 & 0 & $v_{17}$ & $c_{7}$ & $-c_{9}$ & $-c_{0}$ & $v_{32}$ & 0 & $-c_{1}$ & $c_{6}$ \\
$v_{3}$ & $-c_{8}$ & 0 & 0 & $v_{18}$ & $-c_{7}$ & $c_{9}$ & $c_{0}$ & $v_{33}$ & 0 & $-c_{1}$ & $-c_{6}$ \\
$v_{4}$ & 0 & $c_{8}$ & 0 & $v_{19}$ & $-c_{7}$ & $c_{9}$ & $-c_{0}$ & $v_{34}$ & $c_{6}$ & 0 & $c_{1}$ \\
$v_{5}$ & 0 & $-c_{8}$ & 0 & $v_{2}$ & $-c_{7}$ & $-c_{9}$ & $c_{0}$ & $v_{35}$ & $c_{6}$ & 0 & $-c_{1}$ \\
$v_{6}$ & $c_{9}$ & $c_{0}$ & $c_{7}$ & $v_{21}$ & $-c_{7}$ & $-c_{9}$ & $-c_{0}$ & $v_{36}$ & $-c_{6}$ & 0 & $c_{1}$ \\
$v_{7}$ & $c_{9}$ & $c_{0}$ & $-c_{7}$ & $v_{22}$ & $c_{0}$ & $c_{7}$ & $c_{9}$ & $v_{37}$ & $-c_{6}$ & 0 & $-c_{1}$ \\
$v_{8}$ & $c_{9}$ & $-c_{0}$ & $c_{7}$ & $v_{23}$ & $c_{0}$ & $c_{7}$ & $-c_{9}$ & $v_{38}$ & $c_{1}$ & $c_{6}$ & 0 \\
$v_{9}$ & $c_{9}$ & $-c_{0}$ & $-c_{7}$ & $v_{24}$ & $c_{0}$ & $-c_{7}$ & $c_{9}$ & $v_{39}$ & $c_{1}$ & $-c_{6}$ & 0 \\
$v_{10}$ & $-c_{9}$ & $c_{0}$ & $c_{7}$ & $v_{25}$ & $c_{0}$ & $-c_{7}$ & $-c_{9}$ & $v_{40}$ & $-c_{1}$ & $c_{6}$ & 0 \\
$v_{11}$ & $-c_{9}$ & $c_{0}$ & $-c_{7}$ & $v_{26}$ & $-c_{0}$ & $c_{7}$ & $c_{9}$ & $v_{41}$ & $-c_{1}$ & $-c_{6}$ & 0 \\
$v_{12}$ & $-c_{9}$ & $-c_{0}$ & $c_{7}$ & $v_{27}$ & $-c_{0}$ & $c_{7}$ & $-c_{9}$ & $v_{42}$ & $c_{0}$ & $c_{2}$ & $c_{5}$ \\
$v_{13}$ & $-c_{9}$ & $-c_{0}$ & $-c_{7}$ & $v_{28}$ & $-c_{0}$ & $-c_{7}$ & $c_{9}$ & $v_{43}$ & $c_{0}$ & $c_{2}$ & $-c_{5}$ \\
$v_{14}$ & $c_{7}$ & $c_{9}$ & $c_{0}$ & $v_{29}$ & $-c_{0}$ & $-c_{7}$ & $-c_{9}$ & $v_{44}$ & $c_{0}$ & $-c_{2}$ & $c_{5}$
\end{tabular}




\begin{tabular}{|c|c|c|c|c|c|c|c|c|c|c|c|}
\hline & $x$ & $y$ & $z$ & & $x$ & $y$ & $z$ & & $x$ & $y$ & $z$ \\
\hline$v_{45}$ & $c_{0}$ & $-c_{2}$ & $-c_{5}$ & $v_{60}$ & $c_{2}$ & $-c_{5}$ & $c_{0}$ & $v_{75}$ & $c_{4}$ & $c_{3}$ & $-c_{2}$ \\
\hline$v_{46}$ & $-c_{0}$ & $c_{2}$ & $c_{5}$ & $v_{61}$ & $c_{2}$ & $-c_{5}$ & $-c_{0}$ & $v_{76}$ & $c_{4}$ & $-c_{3}$ & $c_{2}$ \\
\hline$v_{47}$ & $-c_{0}$ & $c_{2}$ & $-c_{5}$ & $v_{62}$ & $-c_{2}$ & $c_{5}$ & $c_{0}$ & $v_{77}$ & $c_{4}$ & $-c_{3}$ & $-c_{2}$ \\
\hline$v_{48}$ & $-c_{0}$ & $-c_{2}$ & $c_{5}$ & $v_{63}$ & $-c_{2}$ & $c_{5}$ & $-c_{0}$ & $v_{78}$ & $-c_{4}$ & $c_{3}$ & $c_{2}$ \\
\hline$v_{49}$ & $-c_{0}$ & $-c_{2}$ & $-c_{5}$ & $v_{64}$ & $-c_{2}$ & $-c_{5}$ & $c_{0}$ & $v_{79}$ & $-c_{4}$ & $c_{3}$ & $-c_{2}$ \\
\hline$v_{50}$ & $c_{5}$ & $c_{0}$ & $c_{2}$ & $v_{65}$ & $-c_{2}$ & $-c_{5}$ & $-c_{0}$ & $v_{80}$ & $-c_{4}$ & $-c_{3}$ & $c_{2}$ \\
\hline$v_{51}$ & $c_{5}$ & $c_{0}$ & $-c_{2}$ & $v_{66}$ & $c_{3}$ & $c_{2}$ & $c_{4}$ & $v_{81}$ & $-c_{4}$ & $-c_{3}$ & $-c_{2}$ \\
\hline$v_{52}$ & $c_{5}$ & $-c_{0}$ & $c_{2}$ & $v_{67}$ & $c_{3}$ & $c_{2}$ & $-c_{4}$ & $v_{82}$ & $c_{2}$ & $c_{4}$ & $c_{3}$ \\
\hline$v_{53}$ & $c_{5}$ & $-c_{0}$ & $-c_{2}$ & $v_{68}$ & $c_{3}$ & $-c_{2}$ & $c_{4}$ & $v_{83}$ & $c_{2}$ & $c_{4}$ & $-c_{3}$ \\
\hline$v_{54}$ & $-c_{5}$ & $c_{0}$ & $c_{2}$ & $v_{69}$ & $c_{3}$ & $-c_{2}$ & $-c_{4}$ & $v_{84}$ & $c_{2}$ & $-c_{4}$ & $c_{3}$ \\
\hline$v_{55}$ & $-c_{5}$ & $c_{0}$ & $-c_{2}$ & $v_{70}$ & $-c_{3}$ & $c_{2}$ & $c_{4}$ & $v_{85}$ & $c_{2}$ & $-c_{4}$ & $-c_{3}$ \\
\hline$v_{56}$ & $-c_{5}$ & $-c_{0}$ & $c_{2}$ & $v_{71}$ & $-c_{3}$ & $c_{2}$ & $-c_{4}$ & $v_{86}$ & $-c_{2}$ & $c_{4}$ & $c_{3}$ \\
\hline$v_{57}$ & $-c_{5}$ & $-c_{0}$ & $-c_{2}$ & $v_{72}$ & $-c_{3}$ & $-c_{2}$ & $c_{4}$ & $v_{87}$ & $-c_{2}$ & $c_{4}$ & $-c_{3}$ \\
\hline$v_{58}$ & $c_{2}$ & $c_{5}$ & $c_{0}$ & $v_{73}$ & $-c_{3}$ & $-c_{2}$ & $-c_{4}$ & $v_{88}$ & $-c_{2}$ & $-c_{4}$ & $c_{3}$ \\
\hline$v_{59}$ & $c_{2}$ & $c_{5}$ & $-c_{0}$ & $v_{74}$ & $c_{4}$ & $c_{3}$ & $c_{2}$ & $v_{89}$ & $-c_{2}$ & $-c_{4}$ & $-c_{3}$ \\
\hline
\end{tabular}

The normal vectors $\vec{N}=(A, B, C)$ of surface plane equations passing the corner points given above: Let be

$$
\begin{aligned}
& \lambda_{0}=\frac{-1+\sqrt{5}}{2}, \lambda_{1}=\frac{-5+3 \sqrt{5}}{10}, \lambda_{2}=\frac{-5+4 \sqrt{5}}{11}, \lambda_{3}=\frac{5+7 \sqrt{5}}{22}, \lambda_{4}=\frac{15-\sqrt{5}}{22}, \lambda_{5}=\frac{\sqrt{5}}{5}, \lambda_{6}=\frac{2 \sqrt{5}}{5}, \\
& \lambda_{7}=\frac{5-\sqrt{5}}{5} \text { and } \lambda_{8}=\frac{5+\sqrt{5}}{10} ;
\end{aligned}
$$

\begin{tabular}{|c|c|c|c|c|c|c|c|}
\hline Corner Points & $A$ & $B$ & $C$ & Corner Points & $A$ & $B$ & $C$ \\
\hline $\begin{array}{c}v_{0}, v_{8}, v_{44}, v_{68}, v_{52} \\
v_{34}, v_{50}, v_{66}, v_{42}, v_{6}\end{array}$ & $\lambda_{0}$ & 0 & 1 & $\begin{array}{c}v_{3}, v_{18}, v_{54}, v_{78}, v_{62} \\
v_{40}, v_{63}, v_{79}, v_{55}, v_{19}\end{array}$ & -1 & $\lambda_{0}$ & 0 \\
\hline $\begin{array}{c}v_{0}, v_{10}, v_{46}, v_{70}, v_{54} \\
v_{36}, v_{56}, v_{72}, v_{48}, v_{12}\end{array}$ & $-\lambda_{0}$ & 0 & 1 & $\begin{array}{c}v_{3}, v_{21}, v_{57}, v_{81}, v_{65} \\
v_{41}, v_{64}, v_{80}, v_{56}, v_{20}\end{array}$ & -1 & $-\lambda_{0}$ & 0 \\
\hline $\begin{array}{c}v_{1}, v_{7}, v_{43}, v_{67}, v_{51} \\
v_{35}, v_{53}, v_{69}, v_{45}, v_{9}\end{array}$ & $\lambda_{0}$ & 0 & -1 & $\begin{array}{c}v_{4}, v_{23}, v_{59}, v_{83}, v_{43} \\
v_{31}, v_{47}, v_{87}, v_{63}, v_{27}\end{array}$ & 0 & 1 & $-\lambda_{0}$ \\
\hline $\begin{array}{c}v_{1}, v_{13}, v_{49}, v_{73}, v_{57} \\
v_{37}, v_{55}, v_{71}, v_{47}, v_{11}\end{array}$ & $-\lambda_{0}$ & 0 & -1 & $\begin{array}{c}v_{4}, v_{26}, v_{62}, v_{86}, v_{46} \\
v_{30}, v_{42}, v_{82}, v_{58}, v_{22}\end{array}$ & 0 & 1 & $\lambda_{0}$ \\
\hline $\begin{array}{c}v_{2}, v_{15}, v_{51}, v_{75}, v_{59} \\
v_{38}, v_{58}, v_{74}, v_{50}, v_{14}\end{array}$ & 1 & $\lambda_{0}$ & 0 & $\begin{array}{c}v_{5}, v_{24}, v_{60}, v_{84}, v_{44} \\
v_{32}, v_{48}, v_{88}, v_{64}, v_{28}\end{array}$ & 0 & -1 & $\lambda_{0}$ \\
\hline $\begin{array}{c}v_{2}, v_{16}, v_{52}, v_{76}, v_{60} \\
v_{39}, v_{61}, v_{77}, v_{53}, v_{17}\end{array}$ & 1 & $-\lambda_{0}$ & 0 & $\begin{array}{c}v_{5}, v_{29}, v_{65}, v_{89}, v_{49} \\
v_{33}, v_{45}, v_{85}, v_{61}, v_{25}\end{array}$ & 0 & -1 & $-\lambda_{0}$ \\
\hline$v_{0}, v_{6}, v_{10}$ & 0 & $\lambda_{1}$ & 1 & $v_{3}, v_{19}, v_{21}$ & -1 & 0 & $-\lambda_{1}$ \\
\hline$v_{0}, v_{12}, v_{8}$ & 0 & $-\lambda_{1}$ & 1 & $v_{3}, v_{20}, v_{18}$ & -1 & 0 & $\lambda_{1}$ \\
\hline$v_{1}, v_{9}, v_{13}$ & 0 & $-\lambda_{1}$ & -1 & $v_{4}, v_{22}, v_{23}$ & $\lambda_{1}$ & 1 & 0 \\
\hline$v_{1}, v_{11}, v_{7}$ & 0 & $\lambda_{1}$ & -1 & $v_{4}, v_{27}, v_{26}$ & $-\lambda_{1}$ & 1 & 0 \\
\hline$v_{2}, v_{14}, v_{16}$ & 1 & 0 & $\lambda_{1}$ & $v_{5}, v_{25}, v_{24}$ & $\lambda_{1}$ & -1 & 0 \\
\hline$v_{2}, v_{17}, v_{15}$ & 1 & 0 & $-\lambda_{1}$ & $v_{5}, v_{28}, v_{29}$ & $-\lambda_{1}$ & -1 & 0 \\
\hline$v_{6}, v_{30}, v_{10}$ & 0 & $\lambda_{2}$ & $\lambda_{3}$ & $v_{18}, v_{20}, v_{36}$ & $-\lambda_{3}$ & 0 & $\lambda_{2}$ \\
\hline$v_{7}, v_{11}, v_{31}$ & 0 & $\lambda_{2}$ & $-\lambda_{3}$ & $v_{19}, v_{21}, v_{37}$ & $-\lambda_{3}$ & 0 & $-\lambda_{2}$ \\
\hline$v_{8}, v_{12}, v_{32}$ & 0 & $-\lambda_{2}$ & $\lambda_{3}$ & $v_{22}, v_{38}, v_{23}$ & $\lambda_{2}$ & $\lambda_{3}$ & 0 \\
\hline$v_{9}, v_{13}, v_{33}$ & 0 & $-\lambda_{2}$ & $-\lambda_{3}$ & $v_{24}, v_{25}, v_{39}$ & $\lambda_{2}$ & $-\lambda_{3}$ & 0 \\
\hline$v_{14}, v_{16}, v_{34}$ & $\lambda_{3}$ & 0 & $\lambda_{2}$ & $v_{26}, v_{27}, v_{40}$ & $-\lambda_{2}$ & $\lambda_{3}$ & 0 \\
\hline$v_{15}, v_{17}, v_{35}$ & $\lambda_{3}$ & 0 & $-\lambda_{2}$ & $v_{28}, v_{41}, v_{29}$ & $-\lambda_{2}$ & $-\lambda_{3}$ & 0 \\
\hline$v_{66}, v_{74}, v_{82}$ & $-\lambda_{4}$ & $-\lambda_{4}$ & $-\lambda_{4}$ & $v_{70}, v_{86}, v_{78}$ & $\lambda_{4}$ & $-\lambda_{4}$ & $-\lambda_{4}$ \\
\hline$v_{67}, v_{83}, v_{75}$ & $-\lambda_{4}$ & $-\lambda_{4}$ & $\lambda_{4}$ & $v_{71}, v_{79}, v_{87}$ & $\lambda_{4}$ & $-\lambda_{4}$ & $\lambda_{4}$ \\
\hline$v_{68}, v_{84}, v_{76}$ & $-\lambda_{4}$ & $\lambda_{4}$ & $-\lambda_{4}$ & $v_{72}, v_{80}, v_{88}$ & $\lambda_{4}$ & $\lambda_{4}$ & $-\lambda_{4}$ \\
\hline$v_{69}, v_{77}, v_{85}$ & $-\lambda_{4}$ & $\lambda_{4}$ & $\lambda_{4}$ & $v_{73}, v_{89}, v_{81}$ & $\lambda_{4}$ & $\lambda_{4}$ & $\lambda_{4}$ \\
\hline
\end{tabular}




\begin{tabular}{|c|c|c|c|c|c|c|c|}
\hline Corner Points & $A$ & $B$ & $C$ & Corner Points & $A$ & $B$ & C \\
\hline$v_{6}, v_{42}, v_{30}$ & $\lambda_{1}$ & $\lambda_{5}$ & $\lambda_{6}$ & $v_{18}, v_{36}, v_{54}$ & $-\lambda_{6}$ & $\lambda_{1}$ & $\lambda_{5}$ \\
\hline$v_{7}, v_{43}, v_{31}$ & $\lambda_{1}$ & $\lambda_{5}$ & $-\lambda_{6}$ & $v_{19}, v_{55}, v_{37}$ & $-\lambda_{6}$ & $\lambda_{1}$ & $-\lambda_{5}$ \\
\hline$v_{8}, v_{32}, v_{44}$ & $\lambda_{1}$ & $-\lambda_{5}$ & $\lambda_{6}$ & $v_{20}, v_{56}, v_{36}$ & $-\lambda_{6}$ & $-\lambda_{1}$ & $\lambda_{5}$ \\
\hline$v_{9}, v_{45}, v_{33}$ & $\lambda_{1}$ & $-\lambda_{5}$ & $-\lambda_{6}$ & $v_{21}, v_{37}, v_{57}$ & $-\lambda_{6}$ & $-\lambda_{1}$ & $-\lambda_{5}$ \\
\hline$v_{10}, v_{30}, v_{46}$ & $-\lambda_{1}$ & $\lambda_{5}$ & $\lambda_{6}$ & $v_{22}, v_{58}, v_{38}$ & $\lambda_{5}$ & $\lambda_{6}$ & $\lambda_{1}$ \\
\hline$v_{11}, v_{47}, v_{31}$ & $-\lambda_{1}$ & $\lambda_{5}$ & $-\lambda_{6}$ & $v_{23}, v_{38}, v_{59}$ & $\lambda_{5}$ & $\lambda_{6}$ & $-\lambda_{1}$ \\
\hline$v_{12}, v_{48}, v_{32}$ & $-\lambda_{1}$ & $-\lambda_{5}$ & $\lambda_{6}$ & $v_{24}, v_{39}, v_{60}$ & $\lambda_{5}$ & $-\lambda_{6}$ & $\lambda_{1}$ \\
\hline$v_{13}, v_{33}, v_{49}$ & $-\lambda_{1}$ & $-\lambda_{5}$ & $-\lambda_{6}$ & $v_{25}, v_{61}, v_{39}$ & $\lambda_{5}$ & $-\lambda_{6}$ & $-\lambda_{1}$ \\
\hline$v_{14}, v_{50}, v_{34}$ & $\lambda_{6}$ & $\lambda_{1}$ & $\lambda_{5}$ & $v_{26}, v_{40}, v_{62}$ & $-\lambda_{5}$ & $\lambda_{6}$ & $\lambda_{1}$ \\
\hline$v_{15}, v_{35}, v_{51}$ & $\lambda_{6}$ & $\lambda_{1}$ & $-\lambda_{5}$ & $v_{27}, v_{63}, v_{40}$ & $-\lambda_{5}$ & $\lambda_{6}$ & $-\lambda_{1}$ \\
\hline$v_{16}, v_{34}, v_{52}$ & $\lambda_{6}$ & $-\lambda_{1}$ & $\lambda_{5}$ & $v_{28}, v_{64}, v_{41}$ & $-\lambda_{5}$ & $-\lambda_{6}$ & $\lambda_{1}$ \\
\hline$v_{17}, v_{53}, v_{35}$ & $\lambda_{6}$ & $-\lambda_{1}$ & $-\lambda_{5}$ & $v_{29}, v_{41}, v_{65}$ & $-\lambda_{5}$ & $-\lambda_{6}$ & $-\lambda_{1}$ \\
\hline$v_{42}, v_{66}, v_{82}$ & $\lambda_{5}$ & $-\lambda_{7}$ & $\lambda_{8}$ & $v_{54}, v_{70}, v_{78}$ & $-\lambda_{8}$ & $\lambda_{5}$ & $-\lambda_{7}$ \\
\hline$v_{43}, v_{83}, v_{67}$ & $\lambda_{5}$ & $-\lambda_{7}$ & $-\lambda_{8}$ & $v_{55}, v_{79}, v_{71}$ & $-\lambda_{8}$ & $\lambda_{5}$ & $\lambda_{7}$ \\
\hline$v_{44}, v_{84}, v_{68}$ & $\lambda_{5}$ & $\lambda_{7}$ & $\lambda_{8}$ & $v_{56}, v_{80}, v_{72}$ & $-\lambda_{8}$ & $-\lambda_{5}$ & $-\lambda_{7}$ \\
\hline$v_{45}, v_{69}, v_{85}$ & $\lambda_{5}$ & $\lambda_{7}$ & $-\lambda_{8}$ & $v_{57}, v_{73}, v_{81}$ & $-\lambda_{8}$ & $-\lambda_{5}$ & $\lambda_{7}$ \\
\hline$v_{46}, v_{86}, v_{70}$ & $-\lambda_{5}$ & $-\lambda_{7}$ & $\lambda_{8}$ & $v_{58}, v_{82}, v_{74}$ & $-\lambda_{7}$ & $\lambda_{8}$ & $\lambda_{5}$ \\
\hline$v_{47}, v_{71}, v_{87}$ & $-\lambda_{5}$ & $-\lambda_{7}$ & $-\lambda_{8}$ & $v_{59}, v_{75}, v_{83}$ & $-\lambda_{7}$ & $\lambda_{8}$ & $-\lambda_{5}$ \\
\hline$v_{48}, v_{72}, v_{88}$ & $-\lambda_{5}$ & $\lambda_{7}$ & $\lambda_{8}$ & $v_{60}, v_{76}, v_{84}$ & $-\lambda_{7}$ & $-\lambda_{8}$ & $\lambda_{5}$ \\
\hline$v_{49}, v_{89}, v_{73}$ & $-\lambda_{5}$ & $\lambda_{7}$ & $-\lambda_{8}$ & $v_{61}, v_{85}, v_{77}$ & $-\lambda_{7}$ & $-\lambda_{8}$ & $-\lambda_{5}$ \\
\hline$v_{50}, v_{74}, v_{66}$ & $\lambda_{8}$ & $\lambda_{5}$ & $-\lambda_{7}$ & $v_{62}, v_{78}, v_{86}$ & $\lambda_{7}$ & $\lambda_{8}$ & $\lambda_{5}$ \\
\hline$v_{51}, v_{67}, v_{75}$ & $\lambda_{8}$ & $\lambda_{5}$ & $\lambda_{7}$ & $v_{63}, v_{87}, v_{79}$ & $\lambda_{7}$ & $\lambda_{8}$ & $-\lambda_{5}$ \\
\hline$v_{52}, v_{68}, v_{76}$ & $\lambda_{8}$ & $-\lambda_{5}$ & $-\lambda_{7}$ & $v_{64}, v_{88}, v_{80}$ & $\lambda_{7}$ & $-\lambda_{8}$ & $\lambda_{5}$ \\
\hline$v_{53}, v_{77}, v_{69}$ & $\lambda_{8}$ & $-\lambda_{5}$ & $\lambda_{7}$ & $v_{65}, v_{81}, v_{89}$ & $\lambda_{7}$ & $-\lambda_{8}$ & $-\lambda_{5}$ \\
\hline
\end{tabular}

Surface plane equations of Rectified Truncated Dodecahedron, which normal vectors given in the above tables, can be generalized as follows;

$$
\begin{array}{lll}
\lambda_{0}|x|+|z|=1, & \lambda_{1}|x|+|y|=1, & \lambda_{2}|x|+\lambda_{3}|y|=1 \\
\lambda_{0}|y|+|x|=1, & \lambda_{1}|y|+|z|=1, & \lambda_{2}|y|+\lambda_{3}|z|=1 \\
\lambda_{0}|z|+|y|=1, & \lambda_{1}|z|+|x|=1, & \lambda_{2}|z|+\lambda_{3}|x|=1 \\
\lambda_{1}|x|+\lambda_{5}|y|+\lambda_{6}|z|=1, & \lambda_{1}|y|+\lambda_{5}|z|+\lambda_{6}|x|=1, & \lambda_{1}|z|+\lambda_{5}|x|+\lambda_{6}|y|=1 \\
\lambda_{8}|x|+\lambda_{7}|z|+\lambda_{5}|y|=1, & \lambda_{8}|z|+\lambda_{7}|x|+\lambda_{5}|y|=1, & \lambda_{8}|z|+\lambda_{7}|y|+\lambda_{5}|x|=1 \\
\lambda_{4}|x|+\lambda_{4}|y|+\lambda_{4}|z|=1 & &
\end{array}
$$

Thus, we define the distance function which the unit sphere of analytical space furnishing by this metric is Rectified Truncated Dodecahedron containing all these surface plane equations. This distance function will be denoted by $d_{\text {RTD }}$.

Definition 2.3. Let $X=\left(x_{1}, y_{1}, z_{1}\right)$ and $Y=\left(x_{2}, y_{2}, z_{2}\right)$ be any two points in the $\mathbb{R}^{3}$, for brevity, $\mathbf{X}_{12}=\left|x_{1}-x_{2}\right|$, $\mathbf{Y}_{12}=\left|y_{1}-y_{2}\right|$ and $\mathbf{Z}_{12}=\left|z_{1}-z_{2}\right|$. Then $d_{\mathbf{R T D}}: \mathbb{R}^{3} \times \mathbb{R}^{3} \rightarrow[0, \infty)$ is defined by

$$
d_{\mathbf{R T D}}(X, Y)=\max \left\{F_{i}: i=1, \ldots, 16\right\}
$$

where

$$
\begin{array}{lll}
F_{1}=\lambda_{0} \mathbf{X}_{12}+\mathbf{Z}_{12}, & F_{4}=\lambda_{1} \mathbf{X}_{12}+\mathbf{Y}_{12}, & F_{7}=\lambda_{2} \mathbf{X}_{12}+\lambda_{3} \mathbf{Y}_{12} \\
F_{2}=\lambda_{0} \mathbf{Y}_{12}+\mathbf{X}_{12}, & F_{5}=\lambda_{1} \mathbf{Y}_{12}+\mathbf{Z}_{12}, & F_{8}=\lambda_{2} \mathbf{Y}_{12}+\lambda_{3} \mathbf{Z}_{12} \\
F_{3}=\lambda_{0} \mathbf{Z}_{12}+\mathbf{Y}_{12}, & F_{6}=\lambda_{1} \mathbf{Z}_{12}+\mathbf{X}_{12}, & F_{9}=\lambda_{2} \mathbf{Z}_{12}+\lambda_{3} \mathbf{X}_{12} \\
F_{10}=\lambda_{4} \mathbf{X}_{12}+\lambda_{4} \mathbf{Y}_{12}+\lambda_{4} \mathbf{Z}_{12}, & F_{13}=\lambda_{1} \mathbf{Z}_{12}+\lambda_{5} \mathbf{X}_{12}+\lambda_{6} \mathbf{Y}_{12}, & F_{16}=\lambda_{8} \mathbf{Z}_{12}+\lambda_{7} \mathbf{Y}_{12}+\lambda_{5} \mathbf{X}_{12} \\
F_{11}=\lambda_{1} \mathbf{X}_{12}+\lambda_{5} \mathbf{Y}_{12}+\lambda_{6} \mathbf{Z}_{12}, & F_{14}=\lambda_{8} \mathbf{X}_{12}+\lambda_{7} \mathbf{Z}_{12}+\lambda_{5} \mathbf{Y}_{12}, & \\
F_{12}=\lambda_{1} \mathbf{Y}_{12}+\lambda_{5} \mathbf{Z}_{12}+\lambda_{6} \mathbf{X}_{12}, & F_{15}=\lambda_{8} \mathbf{Y}_{12}+\lambda_{7} \mathbf{X}_{12}+\lambda_{5} \mathbf{Z}_{12}, &
\end{array}
$$

such that $\lambda_{0}=\frac{-1+\sqrt{5}}{2}, \lambda_{1}=\frac{-5+3 \sqrt{5}}{10}, \lambda_{2}=\frac{-5+4 \sqrt{5}}{11}, \lambda_{3}=\frac{5+7 \sqrt{5}}{22}, \lambda_{4}=\frac{15-\sqrt{5}}{22}, \lambda_{5}=\frac{\sqrt{5}}{5}$, $\lambda_{6}=\frac{2 \sqrt{5}}{5}, \lambda_{7}=\frac{5-\sqrt{5}}{5}$ and $\lambda_{8}=\frac{5+\sqrt{5}}{10}$. 


\subsection{Rectified Truncated Icosahedron}

The rectified truncated icosahedron is a polyhedron. It has 92 faces: 60 isosceles triangles, 12 regular pentagons, and 20 regular hexagons. It is constructed as a rectified truncated icosahedron, rectification truncating vertices down to mid-edges. As a near-miss Johnson solid, under icosahedral symmetry, the pentagons are always regular, although the hexagons, while having equal edge lengths, do not have the same edge lengths with the pentagons, having slightly different but alternating angles, causing the triangles to be isosceles instead.

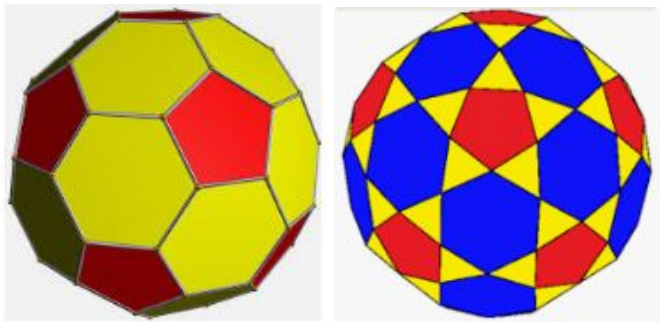

Figure 5: Truncated Icosahedron and Rectified Truncated Icosahedron

Corner Points of Rectified Truncated Icosahedron: Let $c_{0}=\frac{1+\sqrt{5}}{12}, c_{1}=\frac{-1+\sqrt{5}}{4}, c_{2}=\frac{-1+2 \sqrt{5}}{6}, c_{3}=\frac{\sqrt{5}}{3}$, $c_{4}=\frac{7+\sqrt{5}}{12}, c_{5}=\frac{1+\sqrt{5}}{4}, c_{6}=\frac{9+\sqrt{5}}{12}, c_{7}=\frac{1}{6}, c_{8}=\frac{1}{2}$ and $c_{9}=\frac{2}{3}$ any corner point of Rectified Truncated Icosahedron be $v_{i}=(x, y, z)$ for $i=0, \ldots, 89$;

\begin{tabular}{|c|c|c|c|c|c|c|c|c|c|c|c|}
\hline & $x$ & $y$ & $z$ & & $x$ & $y$ & $z$ & & $x$ & $y$ & $z$ \\
\hline$v_{0}$ & 0 & 0 & 1 & $v_{15}$ & $c_{6}$ & $c_{1}$ & $-c_{7}$ & $v_{30}$ & $c_{1}$ & $c_{8}$ & $c_{5}$ \\
\hline$v_{1}$ & 0 & 0 & -1 & $v_{16}$ & $c_{6}$ & $-c_{1}$ & $c_{7}$ & $v_{31}$ & $c_{1}$ & $c_{8}$ & $-c_{5}$ \\
\hline$v_{2}$ & 1 & 0 & 0 & $v_{17}$ & $c_{6}$ & $-c_{1}$ & $-c_{7}$ & $v_{32}$ & $c_{1}$ & $-c_{8}$ & $c_{5}$ \\
\hline$v_{3}$ & -1 & 0 & 0 & $v_{18}$ & $-c_{6}$ & $c_{1}$ & $c_{7}$ & $v_{33}$ & $c_{1}$ & $-c_{8}$ & $-c_{5}$ \\
\hline$v_{4}$ & 0 & 1 & 0 & $v_{19}$ & $-c_{6}$ & $c_{1}$ & $-c_{7}$ & $v_{34}$ & $-c_{1}$ & $c_{8}$ & $c_{5}$ \\
\hline$v_{5}$ & 0 & -1 & 0 & $v_{20}$ & $-c_{6}$ & $-c_{1}$ & $c_{7}$ & $v_{35}$ & $-c_{1}$ & $c_{8}$ & $-c_{5}$ \\
\hline$v_{6}$ & $c_{1}$ & $c_{7}$ & $c_{6}$ & $v_{21}$ & $-c_{6}$ & $-c_{1}$ & $-c_{7}$ & $v_{36}$ & $-c_{1}$ & $-c_{8}$ & $c_{5}$ \\
\hline$v_{7}$ & $c_{1}$ & $c_{7}$ & $-c_{6}$ & $v_{22}$ & $c_{7}$ & $c_{6}$ & $c_{1}$ & $v_{37}$ & $-c_{1}$ & $-c_{8}$ & $-c_{5}$ \\
\hline$v_{8}$ & $c_{1}$ & $-c_{7}$ & $c_{6}$ & $v_{23}$ & $c_{7}$ & $c_{6}$ & $-c_{1}$ & $v_{38}$ & $c_{5}$ & $c_{1}$ & $c_{8}$ \\
\hline$v_{9}$ & $c_{1}$ & $-c_{7}$ & $-c_{6}$ & $v_{24}$ & $c_{7}$ & $-c_{6}$ & $c_{1}$ & $v_{39}$ & $c_{5}$ & $c_{1}$ & $-c_{8}$ \\
\hline$v_{10}$ & $-c_{1}$ & $c_{7}$ & $c_{6}$ & $v_{25}$ & $c_{7}$ & $-c_{6}$ & $-c_{1}$ & $v_{40}$ & $c_{5}$ & $-c_{1}$ & $c_{8}$ \\
\hline$v_{11}$ & $-c_{1}$ & $c_{7}$ & $-c_{6}$ & $v_{26}$ & $-c_{7}$ & $c_{6}$ & $c_{1}$ & $v_{41}$ & $c_{5}$ & $-c_{1}$ & $-c_{8}$ \\
\hline$v_{12}$ & $-c_{1}$ & $-c_{7}$ & $c_{6}$ & $v_{27}$ & $-c_{7}$ & $c_{6}$ & $-c_{1}$ & $v_{42}$ & $-c_{5}$ & $c_{1}$ & $c_{8}$ \\
\hline$v_{13}$ & $-c_{1}$ & $-c_{7}$ & $-c_{6}$ & $v_{28}$ & $-c_{7}$ & $-c_{6}$ & $c_{1}$ & $v_{43}$ & $-c_{5}$ & $c_{1}$ & $-c_{8}$ \\
\hline$v_{14}$ & $c_{6}$ & $c_{1}$ & $c_{7}$ & $v_{29}$ & $-c_{7}$ & $-c_{6}$ & $-c_{1}$ & $v_{44}$ & $-c_{5}$ & $-c_{1}$ & $c_{8}$ \\
\hline$v_{45}$ & $-c_{5}$ & $-c_{1}$ & $-c_{8}$ & $v_{60}$ & $-c_{2}$ & $-c_{0}$ & $c_{4}$ & $v_{75}$ & $-c_{0}$ & $c_{4}$ & $-c_{2}$ \\
\hline$v_{46}$ & $c_{8}$ & $c_{5}$ & $c_{1}$ & $v_{61}$ & $-c_{2}$ & $-c_{0}$ & $-c_{4}$ & $v_{76}$ & $-c_{0}$ & $-c_{4}$ & $c_{2}$ \\
\hline$v_{47}$ & $c_{8}$ & $c_{5}$ & $-c_{1}$ & $v_{62}$ & $c_{4}$ & $c_{2}$ & $c_{0}$ & $v_{77}$ & $-c_{0}$ & $-c_{4}$ & $-c_{2}$ \\
\hline$v_{48}$ & $c_{8}$ & $-c_{5}$ & $c_{1}$ & $v_{63}$ & $c_{4}$ & $c_{2}$ & $-c_{0}$ & $v_{78}$ & 0 & $c_{9}$ & $c_{3}$ \\
\hline$v_{49}$ & $c_{8}$ & $-c_{5}$ & $-c_{1}$ & $v_{64}$ & $c_{4}$ & $-c_{2}$ & $c_{0}$ & $v_{79}$ & 0 & $c_{9}$ & $-c_{3}$ \\
\hline$v_{50}$ & $-c_{8}$ & $c_{5}$ & $c_{1}$ & $v_{65}$ & $c_{4}$ & $-c_{2}$ & $-c_{0}$ & $v_{80}$ & 0 & $-c_{9}$ & $c_{3}$ \\
\hline$v_{51}$ & $-c_{8}$ & $c_{5}$ & $-c_{1}$ & $v_{66}$ & $-c_{4}$ & $c_{2}$ & $c_{0}$ & $v_{81}$ & 0 & $-c_{9}$ & $-c_{3}$ \\
\hline$v_{52}$ & $-c_{8}$ & $-c_{5}$ & $c_{1}$ & $v_{67}$ & $-c_{4}$ & $c_{2}$ & $-c_{0}$ & $v_{82}$ & $c_{3}$ & 0 & $c_{9}$ \\
\hline$v_{53}$ & $-c_{8}$ & $-c_{5}$ & $-c_{1}$ & $v_{68}$ & $-c_{4}$ & $-c_{2}$ & $c_{0}$ & $v_{83}$ & $c_{3}$ & 0 & $-c_{9}$ \\
\hline$v_{54}$ & $c_{2}$ & $c_{0}$ & $c_{4}$ & $v_{69}$ & $-c_{4}$ & $-c_{2}$ & $-c_{0}$ & $v_{84}$ & $-c_{3}$ & 0 & $c_{9}$ \\
\hline$v_{55}$ & $c_{2}$ & $c_{0}$ & $-c_{4}$ & $v_{70}$ & $c_{0}$ & $c_{4}$ & $c_{2}$ & $v_{85}$ & $-c_{3}$ & 0 & $-c_{9}$ \\
\hline$v_{56}$ & $c_{2}$ & $-c_{0}$ & $c_{4}$ & $v_{71}$ & $c_{0}$ & $c_{4}$ & $-c_{2}$ & $v_{86}$ & $c_{9}$ & $c_{3}$ & 0 \\
\hline$v_{57}$ & $c_{2}$ & $-c_{0}$ & $-c_{4}$ & $v_{72}$ & $c_{0}$ & $-c_{4}$ & $c_{2}$ & $v_{87}$ & $c_{9}$ & $-c_{3}$ & 0 \\
\hline$v_{58}$ & $-c_{2}$ & $c_{0}$ & $c_{4}$ & $v_{73}$ & $c_{0}$ & $-c_{4}$ & $-c_{2}$ & $v_{88}$ & $-c_{9}$ & $c_{3}$ & 0 \\
\hline$v_{59}$ & $-c_{2}$ & $c_{0}$ & $-c_{4}$ & $v_{74}$ & $-c_{0}$ & $c_{4}$ & $c_{2}$ & $v_{89}$ & $-c_{9}$ & $-c_{3}$ & 0 \\
\hline
\end{tabular}

The normal vectors $\vec{N}=(A, B, C)$ of surface plane equations passing the corner points given above: Let be 


$$
\begin{aligned}
& \lambda_{0}=\frac{-1+\sqrt{5}}{6}, \lambda_{1}=\frac{3-\sqrt{5}}{2}, \lambda_{2}=\frac{-1+\sqrt{5}}{2}, \lambda_{3}=\frac{27+3 \sqrt{5}}{38}, \lambda_{4}=\frac{-3+6 \sqrt{5}}{19}, \lambda_{5}=\frac{1}{3}, \lambda_{6}=\frac{3+\sqrt{5}}{6} \text { and } \\
& \lambda_{7}=\frac{\sqrt{5}}{3}
\end{aligned}
$$

\begin{tabular}{|c|c|c|c|c|c|c|c|}
\hline Corner Points & $A$ & $B$ & C & Corner Points & $A$ & $B$ & $C$ \\
\hline$v_{78}, v_{34}, v_{10}, v_{0}, v_{6}, v_{30}$ & 0 & $\lambda_{1}$ & 1 & $v_{84}, v_{42}, v_{18}, v_{3}, v_{20}, v_{44}$ & -1 & 0 & $\lambda_{1}$ \\
\hline$v_{79}, v_{31}, v_{7}, v_{1}, v_{11}, v_{35}$ & 0 & $\lambda_{1}$ & -1 & $v_{85}, v_{45}, v_{21}, v_{3}, v_{19}, v_{43}$ & -1 & 0 & $-\lambda_{1}$ \\
\hline$v_{80}, v_{32}, v_{8}, v_{0}, v_{12}, v_{36}$ & 0 & $-\lambda_{1}$ & 1 & $v_{86}, v_{47}, v_{23}, v_{4}, v_{22}, v_{46}$ & $\lambda_{1}$ & 1 & 0 \\
\hline$v_{81}, v_{37}, v_{13}, v_{1}, v_{9}, v_{33}$ & 0 & $-\lambda_{1}$ & -1 & $v_{87}, v_{48}, v_{24}, v_{5}, v_{25}, v_{49}$ & $\lambda_{1}$ & -1 & 0 \\
\hline$v_{82}, v_{40}, v_{16}, v_{2}, v_{14}, v_{38}$ & 1 & 0 & $\lambda_{1}$ & $v_{88}, v_{50}, v_{26}, v_{4}, v_{27}, v_{51}$ & $-\lambda_{1}$ & 1 & 0 \\
\hline$v_{83}, v_{39}, v_{15}, v_{2}, v_{17}, v_{41}$ & 1 & 0 & $-\lambda_{1}$ & $v_{89}, v_{53}, v_{29}, v_{5}, v_{28}, v_{52}$ & $-\lambda_{1}$ & -1 & 0 \\
\hline$v_{0}, v_{8}, v_{6}$ & $\lambda_{0}$ & 0 & 1 & $v_{3}, v_{18}, v_{19}$ & -1 & $\lambda_{0}$ & 0 \\
\hline$v_{0}, v_{10}, v_{12}$ & $-\lambda_{0}$ & 0 & 1 & $v_{3}, v_{21}, v_{20}$ & -1 & $-\lambda_{0}$ & 0 \\
\hline$v_{1}, v_{7}, v_{9}$ & $\lambda_{0}$ & 0 & -1 & $v_{4}, v_{23}, v_{27}$ & 0 & 1 & $-\lambda_{0}$ \\
\hline$v_{1}, v_{13}, v_{11}$ & $-\lambda_{0}$ & 0 & -1 & $v_{4}, v_{26}, v_{22}$ & 0 & 1 & $\lambda_{0}$ \\
\hline$v_{2}, v_{15}, v_{14}$ & 1 & $\lambda_{0}$ & 0 & $v_{5}, v_{24}, v_{28}$ & 0 & -1 & $\lambda_{0}$ \\
\hline$v_{2}, v_{16}, v_{17}$ & 1 & $-\lambda_{0}$ & 0 & $v_{5}, v_{29}, v_{25}$ & 0 & -1 & $-\lambda_{0}$ \\
\hline$v_{70}, v_{30}, v_{54}, v_{38}, v_{62}, v_{46}$ & $\lambda_{2}$ & $\lambda_{2}$ & $\lambda_{2}$ & $v_{74}, v_{50}, v_{66}, v_{42}, v_{58}, v_{34}$ & $-\lambda_{2}$ & $\lambda_{2}$ & $\lambda_{2}$ \\
\hline$v_{71}, v_{47}, v_{63}, v_{39}, v_{55}, v_{31}$ & $\lambda_{2}$ & $\lambda_{2}$ & $-\lambda_{2}$ & $v_{75}, v_{35}, v_{59}, v_{43}, v_{67}, v_{51}$ & $-\lambda_{2}$ & $\lambda_{2}$ & $-\lambda_{2}$ \\
\hline$v_{72}, v_{48}, v_{64}, v_{40}, v_{56}, v_{32}$ & $\lambda_{2}$ & $-\lambda_{2}$ & $\lambda_{2}$ & $v_{76}, v_{36}, v_{60}, v_{44}, v_{68}, v_{52}$ & $-\lambda_{2}$ & $-\lambda_{2}$ & $\lambda_{2}$ \\
\hline$v_{73}, v_{33}, v_{57}, v_{41}, v_{65}, v_{49}$ & $\lambda_{2}$ & $-\lambda_{2}$ & $-\lambda_{2}$ & $v_{77}, v_{53}, v_{69}, v_{45}, v_{61}, v_{37}$ & $-\lambda_{2}$ & $-\lambda_{2}$ & $-\lambda_{2}$ \\
\hline$v_{78}, v_{70}, v_{22}, v_{26}, v_{74}$ & 0 & $\lambda_{3}$ & $\lambda_{4}$ & $v_{84}, v_{60}, v_{12}, v_{10}, v_{58}$ & $-\lambda_{4}$ & 0 & $\lambda_{3}$ \\
\hline$v_{79}, v_{75}, v_{27}, v_{23}, v_{71}$ & 0 & $\lambda_{3}$ & $-\lambda_{4}$ & $v_{85}, v_{59}, v_{11}, v_{13}, v_{61}$ & $-\lambda_{4}$ & 0 & $-\lambda_{3}$ \\
\hline$v_{80}, v_{76}, v_{28}, v_{24}, v_{72}$ & 0 & $-\lambda_{3}$ & $\lambda_{4}$ & $v_{86}, v_{62}, v_{14}, v_{15}, v_{63}$ & $\lambda_{3}$ & $\lambda_{4}$ & 0 \\
\hline$v_{81}, v_{73}, v_{25}, v_{29}, v_{77}$ & 0 & $-\lambda_{3}$ & $-\lambda_{4}$ & $v_{87}, v_{65}, v_{17}, v_{16}, v_{64}$ & $\lambda_{3}$ & $-\lambda_{4}$ & 0 \\
\hline$v_{82}, v_{54}, v_{6}, v_{8}, v_{56}$ & $\lambda_{4}$ & 0 & $\lambda_{3}$ & $v_{88}, v_{67}, v_{19}, v_{18}, v_{66}$ & $-\lambda_{3}$ & $\lambda_{4}$ & 0 \\
\hline$v_{83}, v_{57}, v_{9}, v_{7}, v_{55}$ & $\lambda_{4}$ & 0 & $-\lambda_{3}$ & $v_{89}, v_{68}, v_{20}, v_{21}, v_{69}$ & $-\lambda_{3}$ & $-\lambda_{4}$ & 0 \\
\hline$v_{30}, v_{6}, v_{54}$ & $2 \lambda_{0}$ & $\lambda_{5}$ & $\lambda_{6}$ & $v_{30}, v_{70}, v_{78}$ & $\lambda_{0}$ & $2 \lambda_{5}$ & $\lambda_{7}$ \\
\hline$v_{31}, v_{55}, v_{7}$ & $2 \lambda_{0}$ & $\lambda_{5}$ & $-\lambda_{6}$ & $v_{31}, v_{79}, v_{71}$ & $\lambda_{0}$ & $2 \lambda_{5}$ & $-\lambda_{7}$ \\
\hline$v_{32}, v_{56}, v_{8}$ & $2 \lambda_{0}$ & $-\lambda_{5}$ & $\lambda_{6}$ & $v_{32}, v_{80}, v_{72}$ & $\lambda_{0}$ & $-2 \lambda_{5}$ & $\lambda_{7}$ \\
\hline$v_{33}, v_{9}, v_{57}$ & $2 \lambda_{0}$ & $-\lambda_{5}$ & $-\lambda_{6}$ & $v_{33}, v_{73}, v_{81}$ & $\lambda_{0}$ & $-2 \lambda_{5}$ & $-\lambda_{7}$ \\
\hline$v_{34}, v_{58}, v_{10}$ & $-2 \lambda_{0}$ & $-\lambda_{5}$ & $\lambda_{6}$ & $v_{34}, v_{78}, v_{74}$ & $-\lambda_{0}$ & $2 \lambda_{5}$ & $\lambda_{7}$ \\
\hline$v_{35}, v_{11}, v_{59}$ & $-2 \lambda_{0}$ & $\lambda_{5}$ & $-\lambda_{6}$ & $v_{35}, v_{75}, v_{79}$ & $-\lambda_{0}$ & $2 \lambda_{5}$ & $-\lambda_{7}$ \\
\hline$v_{36}, v_{12}, v_{60}$ & $-2 \lambda_{0}$ & $-\lambda_{5}$ & $\lambda_{6}$ & $v_{36}, v_{76}, v_{80}$ & $-\lambda_{0}$ & $-2 \lambda_{5}$ & $\lambda_{7}$ \\
\hline$v_{37}, v_{61}, v_{13}$ & $-2 \lambda_{0}$ & $-\lambda_{5}$ & $-\lambda_{6}$ & $v_{37}, v_{81}, v_{77}$ & $-\lambda_{0}$ & $-2 \lambda_{5}$ & $-\lambda_{7}$ \\
\hline$v_{38}, v_{14}, v_{62}$ & $\lambda_{6}$ & $2 \lambda_{0}$ & $\lambda_{5}$ & $v_{38}, v_{54}, v_{82}$ & $\lambda_{7}$ & $\lambda_{0}$ & $2 \lambda_{5}$ \\
\hline$v_{39}, v_{63}, v_{15}$ & $\lambda_{6}$ & $2 \lambda_{0}$ & $-\lambda_{5}$ & $v_{39}, v_{83}, v_{55}$ & $\lambda_{7}$ & $\lambda_{0}$ & $-2 \lambda_{5}$ \\
\hline$v_{40}, v_{64}, v_{16}$ & $\lambda_{6}$ & $-2 \lambda_{0}$ & $\lambda_{5}$ & $v_{40}, v_{82}, v_{56}$ & $\lambda_{7}$ & $-\lambda_{0}$ & $2 \lambda_{5}$ \\
\hline$v_{41}, v_{17}, v_{65}$ & $\lambda_{6}$ & $-2 \lambda_{0}$ & $-\lambda_{5}$ & $v_{41}, v_{57}, v_{83}$ & $\lambda_{7}$ & $-\lambda_{0}$ & $-2 \lambda_{5}$ \\
\hline$v_{42}, v_{66}, v_{18}$ & $-\lambda_{6}$ & $2 \lambda_{0}$ & $\lambda_{5}$ & $v_{42}, v_{84}, v_{58}$ & $-\lambda_{7}$ & $\lambda_{0}$ & $2 \lambda_{5}$ \\
\hline$v_{43}, v_{19}, v_{67}$ & $-\lambda_{6}$ & $2 \lambda_{0}$ & $-\lambda_{5}$ & $v_{43}, v_{59}, v_{85}$ & $-\lambda_{7}$ & $\lambda_{0}$ & $-2 \lambda_{5}$ \\
\hline$v_{44}, v_{20}, v_{68}$ & $-\lambda_{6}$ & $-2 \lambda_{0}$ & $\lambda_{5}$ & $v_{44}, v_{60}, v_{84}$ & $-\lambda_{7}$ & $-\lambda_{0}$ & $2 \lambda_{5}$ \\
\hline$v_{45}, v_{69}, v_{21}$ & $-\lambda_{6}$ & $-2 \lambda_{0}$ & $-\lambda_{5}$ & $v_{45}, v_{85}, v_{61}$ & $-\lambda_{7}$ & $-\lambda_{0}$ & $-2 \lambda_{5}$ \\
\hline$v_{46}, v_{22}, v_{70}$ & $\lambda_{5}$ & $\lambda_{6}$ & $2 \lambda_{0}$ & $v_{46}, v_{62}, v_{86}$ & $2 \lambda_{5}$ & $\lambda_{7}$ & $\lambda_{0}$ \\
\hline$v_{47}, v_{71}, v_{23}$ & $\lambda_{5}$ & $\lambda_{6}$ & $-2 \lambda_{0}$ & $v_{47}, v_{86}, v_{63}$ & $2 \lambda_{5}$ & $\lambda_{7}$ & $-\lambda_{0}$ \\
\hline$v_{48}, v_{72}, v_{24}$ & $\lambda_{5}$ & $-\lambda_{6}$ & $2 \lambda_{0}$ & $v_{48}, v_{87}, v_{64}$ & $2 \lambda_{5}$ & $-\lambda_{7}$ & $\lambda_{0}$ \\
\hline$v_{49}, v_{25}, v_{73}$ & $\lambda_{5}$ & $-\lambda_{6}$ & $-2 \lambda_{0}$ & $v_{49}, v_{65}, v_{87}$ & $2 \lambda_{5}$ & $-\lambda_{7}$ & $-\lambda_{0}$ \\
\hline$v_{50}, v_{74}, v_{26}$ & $-\lambda_{5}$ & $\lambda_{6}$ & $2 \lambda_{0}$ & $v_{50}, v_{88}, v_{66}$ & $-2 \lambda_{5}$ & $\lambda_{7}$ & $\lambda_{0}$ \\
\hline$v_{51}, v_{27}, v_{75}$ & $-\lambda_{5}$ & $\lambda_{6}$ & $-2 \lambda_{0}$ & $v_{51}, v_{67}, v_{88}$ & $-2 \lambda_{5}$ & $\lambda_{7}$ & $-\lambda_{0}$ \\
\hline$v_{52}, v_{28}, v_{76}$ & $-\lambda_{5}$ & $-\lambda_{6}$ & $2 \lambda_{0}$ & $v_{52}, v_{68}, v_{89}$ & $-2 \lambda_{5}$ & $-\lambda_{7}$ & $\lambda_{0}$ \\
\hline$v_{53}, v_{77}, v_{29}$ & $-\lambda_{5}$ & $-\lambda_{6}$ & $-2 \lambda_{0}$ & $v_{53}, v_{89}, v_{69}$ & $-2 \lambda_{5}$ & $-\lambda_{7}$ & $-\lambda_{0}$ \\
\hline
\end{tabular}

Surface plane equations of Rectified Truncated Icosahedron, which normal vectors given in the above tables, can be 
generalized as follows;

$$
\begin{array}{lll}
\lambda_{0}|x|+|z|=1, & \lambda_{1}|x|+|y|=1, & \lambda_{3}|x|+\lambda_{4}|y|=1 \\
\lambda_{0}|y|+|x|=1, & \lambda_{1}|y|+|z|=1, & \lambda_{3}|y|+\lambda_{4}|z|=1 \\
\lambda_{0}|z|+|y|=1, & \lambda_{1}|z|+|x|=1, & \lambda_{3}|z|+\lambda_{4}|x|=1 \\
\lambda_{2}(|x|+|y|+|z|)=1, & 2 \lambda_{0}|z|+\lambda_{5}|x|+\lambda_{6}|y|=1, & \lambda_{0}|z|+2 \lambda_{5}|x|+\lambda_{7}|y|=1 \\
2 \lambda_{0}|x|+\lambda_{5}|y|+\lambda_{6}|z|=1, & \lambda_{0}|x|+2 \lambda_{5}|y|+\lambda_{7}|z|=1, & \\
2 \lambda_{0}|y|+\lambda_{5}|z|+\lambda_{6}|x|=1, & \lambda_{0}|y|+2 \lambda_{5}|z|+\lambda_{7}|x|=1, &
\end{array}
$$

Thus, we define the distance function which the unit sphere of analytical space furnishing by this metric is Rectified Truncated Icosahedron containing all these surface plane equations. This distance function will be denoted by $d_{\text {RTI }}$.

Definition 2.4. Let $X=\left(x_{1}, y_{1}, z_{1}\right)$ and $Y=\left(x_{2}, y_{2}, z_{2}\right)$ be any two points in the $\mathbb{R}^{3}$, for brevity, $\mathbf{X}_{12}=\left|x_{1}-x_{2}\right|$, $\mathbf{Y}_{12}=\left|y_{1}-y_{2}\right|$ and $\mathbf{Z}_{12}=\left|z_{1}-z_{2}\right|$. Then $d_{\mathbf{R T I}}: \mathbb{R}^{3} \times \mathbb{R}^{3} \rightarrow[0, \infty)$ is defined by

$$
d_{\mathbf{R T I}}(X, Y)=\max \left\{F_{i}: i=1, \ldots, 16\right\}
$$

where

$$
\begin{array}{lll}
F_{1}=\lambda_{0} \mathbf{X}_{12}+\mathbf{Z}_{12}, & F_{4}=\lambda_{1} \mathbf{X}_{12}+\mathbf{Y}_{12}, & F_{7}=\lambda_{3} \mathbf{X}_{12}+\lambda_{4} \mathbf{Y}_{12} \\
F_{2}=\lambda_{0} \mathbf{Y}_{12}+\mathbf{X}_{12}, & F_{5}=\lambda_{1} \mathbf{Y}_{12}+\mathbf{Z}_{12}, & F_{8}=\lambda_{3} \mathbf{Y}_{12}+\lambda_{4} \mathbf{Z}_{12} \\
F_{3}=\lambda_{0} \mathbf{Z}_{12}+\mathbf{Y}_{12}, & F_{6}=\lambda_{1} \mathbf{Z}_{12}+\mathbf{X}_{12}, & F_{9}=\lambda_{3} \mathbf{Z}_{12}+\lambda_{4} \mathbf{X}_{12} \\
F_{10}=\lambda_{2}\left(\mathbf{X}_{12}+\mathbf{Y}_{12}+\mathbf{Z}_{12}\right), & F_{13}=2 \lambda_{0} \mathbf{Z}_{12}+\lambda_{5} \mathbf{X}_{12}+\lambda_{6} \mathbf{Y}_{12}, & F_{16}=\lambda_{0} \mathbf{Z}_{12}+2 \lambda_{5} \mathbf{X}_{12}+\lambda_{7} \mathbf{Y}_{12} \\
F_{11}=2 \lambda_{0} \mathbf{X}_{12}+\lambda_{5} \mathbf{Y}_{12}+\lambda_{6} \mathbf{Z}_{12}, & F_{14}=\lambda_{0} \mathbf{X}_{12}+2 \lambda_{5} \mathbf{Y}_{12} v \lambda_{7} \mathbf{Z}_{12}, & \\
F_{12}=2 \lambda_{0} \mathbf{Y}_{12}+\lambda_{5} \mathbf{Z}_{12}+\lambda_{6} \mathbf{X}_{12}, & F_{15}=\lambda_{0} \mathbf{Y}_{12}+2 \lambda_{5} \mathbf{Z}_{12}+\lambda_{7} \mathbf{X}_{12}, &
\end{array}
$$

such that $\lambda_{0}=\frac{-1+\sqrt{5}}{6}, \lambda_{1}=\frac{3-\sqrt{5}}{2}, \lambda_{2}=\frac{-1+\sqrt{5}}{2}, \lambda_{3}=\frac{27+3 \sqrt{5}}{38}, \lambda_{4}=\frac{-3+6 \sqrt{5}}{19}, \lambda_{5}=\frac{1}{3}, \lambda_{6}=\frac{3+\sqrt{5}}{6}$ and $\lambda_{7}=\frac{\sqrt{5}}{3}$.

\section{Isometric group of the space $\mathbb{R}^{3}$ according to different metrics}

For the sake of simple, $\mathbb{R}^{3}$ fullfiled by the metrics $d_{\mathbf{R T C}}, d_{\mathbf{R T O}}, d_{\mathbf{R T D}}$ and $d_{\mathbf{R T I}}$ is denoted $\mathbb{R}_{\mathbf{R T C}}^{3}, \mathbb{R}_{\mathbf{R T O}}^{3}, \mathbb{R}_{\mathbf{R T D}}^{3}$ and $\mathbb{R}_{\mathbf{R T I}}^{3}$, respectively, in the rest of the article. Linear structure of these spaces is the same as three dimensional Euclidean space, only the distance function is different.

In this section, we want to answer the question : "What are the isometry groups of the spaces $\mathbb{R}_{\mathrm{RTC}}^{3}, \mathbb{R}_{\mathrm{RTO}}^{3}, \mathbb{R}_{\mathrm{RTD}}^{3}$ and $\mathbb{R}_{\mathrm{RTI}}^{3}$, but we know from the theorem 12 in the reference [13] that if the unit ball $C$ of $(V,\|\cdot\|)$ does not intersect a two-plane in an ellipse, then the group $I(3)$ of isometries of $(V,\|\cdot\|)$ is isomorphic to the semi-direct product of the translation group $T(3)$ of $\mathbb{R}^{3}$ with a finite subgroup of the group of linear transformations with determinant \pm 1 . After this theorem remains a single question. This question is that what is the relevant subgroup?

At the end of this section, we are going to show isometry groups of the spaces $\mathbb{R}_{\mathbf{R T C}}^{3}, \mathbb{R}_{\mathbf{R T O}}^{3}, \mathbb{R}_{\mathbf{R T D}}^{3}$ and $\mathbb{R}_{\mathbf{R T I}}^{3}$ are the semi direct product of " Euclidean symmetry groups of Rectified Archimedean Solids " and " all Euclidean translations of $\mathbb{R}^{3}$ ". Euclidean symmetry group of Rectifed truncated cube and octahedron is the octahedral group $O_{h}$ which is the group of symmetries of the cube and octahedron having order 48. Similarly, Euclidean symmetry group of Rectifed truncated dodecahedron and icosahedron is the icosahedral group $I_{h}$ which is the group of symmetries of the dodecahedron and icosahedron having order 120. Generally, $O_{h}$ and $I_{h}$ consist of identity, reflections, rotations, inversion, rotary reflections and rotary inversions (see details [5], [6], [7], [16]). Before we give isometries of the spaces $\mathbb{R}_{\mathbf{R T C}}^{3}, \mathbb{R}_{\mathbf{R T O}}^{3}, \mathbb{R}_{\mathbf{R T D}}^{3}$ and $\mathbb{R}_{\mathbf{R T I}}^{3}$, we briefly introduce elements of the sets $O_{h}$ and $I_{h}$.

A transformation is any function mapping a set to itself in $\mathbb{R}^{3}$. A figure in $\mathbb{R}^{3}$ is any subset of $\mathbb{R}^{3}$. An isometry of the spaces $\mathbb{R}_{\mathbf{R T C}}^{3}, \mathbb{R}_{\mathbf{R T O}}^{3}, \mathbb{R}_{\mathbf{R T D}}^{3}$ and $\mathbb{R}_{\mathrm{RTI}}^{3}$ is a transformation from $\mathbb{R}^{3}$ onto $\mathbb{R}^{3}$ that preserves distance. This means $d(X, Y)=d(\alpha(X), \alpha(Y))$ for each points $X$ and $Y$ in the spaces $\mathbb{R}_{\mathbf{R T C}}^{3}, \mathbb{R}_{\mathbf{R T O}}^{3}, \mathbb{R}_{\mathbf{R T D}}^{3}$ and $\mathbb{R}_{\mathbf{R T I}}^{3}$, where $\alpha$ is a transformation on these spaces. A symmetry of a figure $F$ in $\mathbb{R}^{3}$ is an isometry mapping $F$ onto itself-that is, an isometry $f: \mathbb{R}^{3} \rightarrow \mathbb{R}^{3}$ such that $f(F)=F$. The identity function $I$ is a transformation is given $I(X)=X$ for each point $X$ in $\mathbb{R}^{3}$. If $\Delta$ represents a plane, then the reflection $\sigma_{\Delta}$ across the plane $\Delta$ fixes every point on $\Delta$, and takes every point $X$ not on $\Delta$ to $Y$, where $\Delta$ is the perpendicular bisector of $X$ and $Y$. A rotation is an isometric transformation which can be written as the composition of two distinc reflections. That is, a rotation about axis $l$ is 
defined by $\sigma_{\Delta} \sigma_{\Gamma}$ where two planes $\Gamma$ and $\Delta$ intersect at line $l$. A rotary reflection is an transformation which is the combination of a rotation about an axis and a reflection in a plane. That is, a rotary reflection is defined by $\sigma_{\Pi} \sigma_{\Delta} \sigma_{\Gamma}$ such that $\Gamma$ and $\Delta$ are two intersecting planes each perpendicular to plane $\Pi$. A inversion according to a point $P$ can be written as the $\sigma_{P}(X)=Y$ such that $P$ is the midpoint of $X$ and $Y$ for $X, Y \in \mathbb{R}^{3}$. Rotary inversion is the combination of a rotation and an inversion in a point.

Definition 3.1. Let $A$ and $B$ be points in $\mathbb{R}_{\mathbf{d}}^{n}$. The minimum distance set of $A, B$ is denoted by $[A B]_{\mathbf{d}}$ and is defined by

$$
[A B]_{\mathbf{d}}=\left\{X \in \mathbb{R}^{n}: \mathbf{d}(A, X)+\mathbf{d}(X, B)=\mathbf{d}(A, B)\right\}
$$

where $\mathbf{d}$ is the metric defined in $\mathbb{R}^{n}$. 6-b),

If the $\mathbf{d}$ metric is taken $d_{\mathbf{R T C}}$, then the minimum distance set $[A B]_{d_{\mathbf{R T C}}}$ is an octagonal dipyramid (see Figure 6-a),

If the $\mathbf{d}$ metric is taken $d_{\mathbf{R T O}}$, then the minimum distance set $[A B]_{d_{\mathbf{R T O}}}$ is a hexagonal dipyramid (see Figure 6-c),

If the $\mathbf{d}$ metric is taken $d_{\mathbf{R T D}}$, then the minimum distance set $[A B]_{d_{\mathrm{RTD}}}$ is a decagonal dipyramid (see Figure 6-a).

If the $\mathbf{d}$ metric is taken $d_{\mathbf{R T I}}$, then the minimum distance set $[A B]_{d_{\mathrm{RTI}}}$ is a hexagonal dipyramid (see Figure
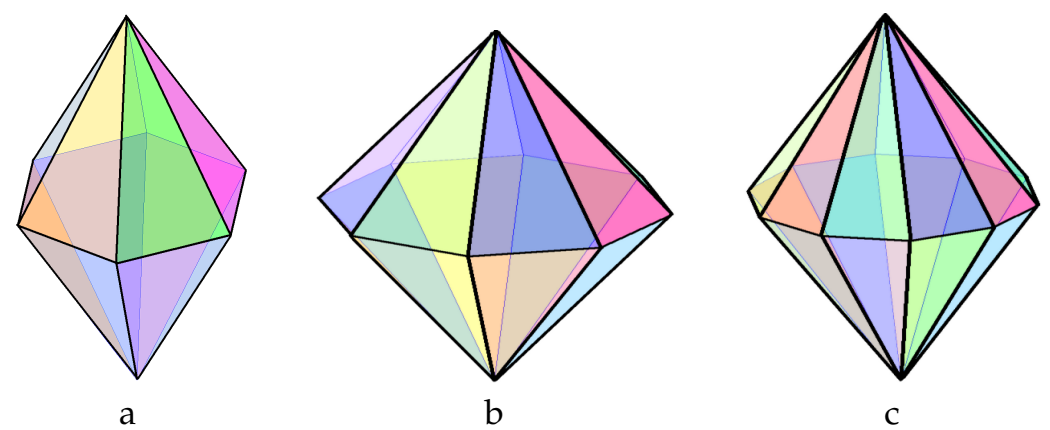

Figure 6

Theorem 3.1. If the function $\phi: \mathbb{R}_{\mathbf{d}}^{3} \rightarrow \mathbb{R}_{\mathbf{d}}^{3}$ is an isometry, then

$$
\phi\left([A B]_{\mathbf{d}}\right)=[\phi(A) \phi(B)]_{\mathbf{d}} .
$$

Proof. Let $Y \in \phi([A B])$. Then,

$$
\begin{aligned}
Y \in \phi\left([A B]_{\mathbf{d}}\right) & \Leftrightarrow \exists X \ni Y=\phi(X) \\
& \Leftrightarrow \mathbf{d}(A, X)+\mathbf{d}(X, B)=\mathbf{d}(A, B) \\
& \Leftrightarrow \mathbf{d}(\phi(A), \phi(X))+\mathbf{d}(\phi(X), \phi(B))=\mathbf{d}(\phi(A), \phi(B)) \\
& \Leftrightarrow Y=\phi(X) \in[\phi(A) \phi(B)]_{\mathbf{d}} .
\end{aligned}
$$

Corollary 3.1. Let the function $\phi: \mathbb{R}_{\mathbf{d}}^{3} \rightarrow \mathbb{R}_{\mathbf{d}}^{3}$ be an isometry. Then the function $\phi$ maps vertices to vertices and preserves the lengths of edges of $[A B]_{\mathbf{d}}$.

Proposition 3.1. Let $f: \mathbb{R}_{\mathbf{d}}^{3} \rightarrow \mathbb{R}_{\mathbf{d}}^{3}$ be an isometry such that $f(O)=O$. Then $f$ is either in $O_{h}$ when $\mathbf{d}=d_{\mathbf{R T C}}$ and $\mathbf{d}=d_{\mathbf{R T O}}$, or in $I_{h}$ when $\mathbf{d}=d_{\mathbf{R T D}}$ and $\mathbf{d}=d_{\mathbf{R T I}}$.

Proof. Consider the minimum distance set $[O P]_{d_{\mathrm{RTC}}}$ for $P=(2,0,0)$. $[O P]_{d_{\mathrm{RTC}}}$ is the octagonal dipyramid whose eight corner points are the corner points $v_{0}, v_{22}, v_{5}, v_{23}, v_{20}, v_{1}, v_{21}, v_{4}$ of the Rectified Truncated Cube with center at origin and radius 1 (see Figure 7-a). The function $\phi$ maps points $v_{0}, v_{22}, v_{5}, v_{23}, v_{20}, v_{1}, v_{21}, v_{4}$ to the vertices of a Rectified Truncated Cube by Corollary 3.1. Since $\phi$ preserve the lengths of edges of the $[O P]_{d_{\mathrm{RTC}}}$, the corner points of the set $\phi\left([O P]_{d_{\mathrm{RTC}}}\right)$ must be on the corner points of octagonal faces of Rectified Truncated Cube. Thus, for each octagonal face of Rectified Truncated Cube, $\phi\left([O P]_{d_{\mathrm{RTC}}}\right)$ can be placed in 8 different ways, and also Rectified 
Truncated Cube has 6 octogonal faces. Consequently, total number of possibilities are $6 \times 8=48$ which implies that the number of ( Euclidean ) symmetries of Rectified Truncated Cube. One can easily show that each of all possibilities is one of the Euclidean symmetries Rectified Truncated Cube.

Consider the minimum distance set $[O Q]_{d_{\mathrm{RTO}}}$, for $Q=(1,1,1) .[O Q]_{d_{\mathrm{RTO}}}$ is the hexagonal dipyramid whose corner points are the corner points $v_{0}, v_{24}, v_{8}, v_{28}, v_{16}, v_{32}$ of the Rectified Truncated Octahedron with center at origin and radius 1 (see Figure 7-b). The function $\phi$ maps points $v_{0}, v_{24}, v_{8}, v_{28}, v_{16}, v_{32}$ to the vertices of a Rectified Truncated Octahedron by Corollary 3.1. Since $\phi$ preserve the lengths of edges the $[O Q]_{d_{\mathrm{RTO}}}$, the corner points of the set $\phi\left([O Q]_{d_{\mathrm{RTO}}}\right)$ must be on the corner points of hexagonal faces of Rectified Truncated Octahedron. Thus, for each hexagonal face of Rectified Truncated Octahedron, $\phi\left([O Q]_{d_{\mathrm{RTO}}}\right)$ can be placed in 6 different ways, and also Rectified Truncated Octahedron has 8 hexagonal faces. Consequently, total number of possibilities are $8 \times 6=48$ which implies that the number of (Euclidean ) symmetries of Rectified Truncated Octahedron. One can easily show that each of all possibilities is one of the Euclidean symmetries Rectified Truncated Octahedron.

Consider the minimum distance set $[O R]_{d_{\mathrm{RTD}}}$ for $R=\left(\frac{5+\sqrt{5}}{5}, \frac{2 \sqrt{5}}{5}, 0\right) .[O R]_{d_{\mathrm{RTD}}}$ is the decagonal dipyramid whose corner points are the corner points $v_{2}, v_{15}, v_{51}, v_{75}, v_{59}, v_{38}, v_{58}, v_{74}, v_{50}, v_{14}$ of the Rectified Truncated Dodecahedron with center at origin and radius 1 (see Figure 7-c). The function $\phi$ maps points $v_{2}, v_{15}, v_{51}, v_{75}, v_{59}, v_{38}$, $v_{58}, v_{74}, v_{50}, v_{14}$ to the vertices of a Rectified Truncated Dodecahedron by Corollary 3.1. Since $\phi$ preserve the lengths of the $[O R]_{d_{\mathrm{RTD}}}$, the corner points of the set $\phi\left([O R]_{d_{\mathrm{RTD}}}\right)$ must be on the corner points of decagononal faces of Rectified Truncated Dodecahedron. Thus, for each decagononal face of Rectified Truncated Dodecahedron, $\phi\left([O R]_{d_{\mathrm{RTD}}}\right)$ can be placed in 12 different ways, and also Rectified Truncated Dodecahedron has 12 decagononal faces. Consequently, total number of possibilities are $10 \times 12=120$ which implies that the number of ( Euclidean ) symmetries of Rectified Truncated Dodecahedron. One can easily show that each of all possibilities is one of the Euclidean symmetries Rectified Truncated Dodecahedron.

Consider the minimum distance set $[O S]_{d_{\mathrm{RTI}}}$, for $S=\left(\frac{3+\sqrt{5}}{3}, 0, \frac{2}{3}\right) \cdot[O S]_{d_{\mathrm{RTI}}}$ is the hexagonal dipyramid whose corner points are the corner points $v_{82}, v_{40}, v_{16}, v_{2}, v_{14}, v_{38}$ of the Rectified Truncated Icosahedron with center at origin and radius 1 (see Figure 7-d). The function $\phi$ maps points $v_{82}, v_{40}, v_{16}, v_{2}, v_{14}, v_{38}$ to the vertices of a Rectified Truncated Icosahedron by Corollary 3.1. Since $\phi$ preserve the lengths of the $[O S]_{d_{\mathrm{RTI}}}$, the corner points of the set $\phi\left([O S]_{d_{\mathrm{RTI}}}\right)$ must be on the corner points of hexagonal faces of Rectified Truncated Icosahedron. Thus, for each octagonal face of Rectified Truncated Icosahedron, $\phi\left([O S]_{d_{\mathrm{RTI}}}\right)$ can be placed in 6 different ways, and also Rectified Truncated Icosahedron has 8 octagonal faces. Consequently, total number of possibilities are $20 \times 6=120$ which implies that the number of ( Euclidean ) symmetries of Rectified Truncated Icosahedron. One can easily show that each of all possibilities is one of the Euclidean symmetries Rectified Truncated Icosahedron.

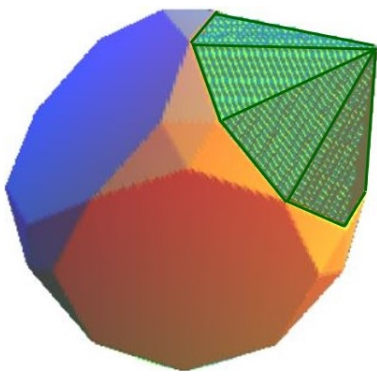

a

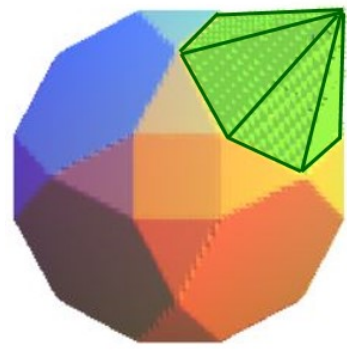

b

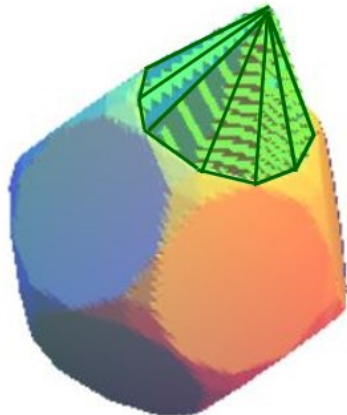

C

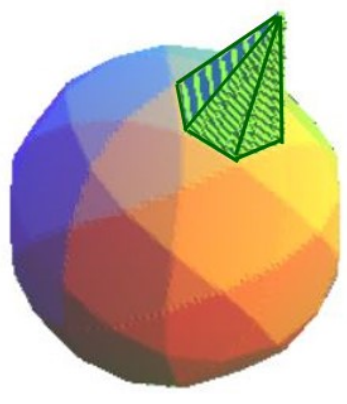

$\mathrm{d}$

Figure 7

Theorem 3.2. Let the function $f: \mathbb{R}_{\mathbf{d}}^{3} \rightarrow \mathbb{R}_{\mathbf{d}}^{3}$ be an isometry. If the $\mathbf{d}$ metric is taken $d_{\mathbf{R T C}}$ and $d_{\mathbf{R T O}}$, then there exists a unique $T_{A} \in T(3)$ and $g \in O_{h}$ such that $f=T_{A} \circ g$. Similarly, If the $\mathbf{d}$ metric is taken $d_{\mathbf{R T D}}$ and $d_{\mathbf{R T I}}$, then there exists a unique $T_{A} \in T(3)$ and $g \in I_{h}$ such that $f=T_{A} \circ g$ 
Proof. Let $f(O)=A$ where $A=\left(a_{1}, a_{2}, a_{3}\right)$. Define $g=T_{-A} \circ f$. We know that $g$ is an isometry and $g(O)=O$. Thus, $g \in O_{h}$ or $g \in I_{h}$ and $f=T_{A} \circ g$ by Proposition 2. The proof of uniqueness is trivial.

\section{Acknowledgments}

This work was supported by the Scientific Research Projects Commission of Eskisehir Osmangazi University under Project Number 201719A235.

\section{References}

[1] Atiyah, M., Sutcliffe, P.: Polyhedra in Physics, Chemistry and Geometry. Milan Journal of Mathematics. 71, 33-58 (2003).

[2] Berger, M.: Geometry I. Springer-Verlag (2004).

[3] Berger, M.: Geometry II, Springer-Verlag (2009).

[4] Carrizales, J.M.M., Lopez, J.L.R., Pal, U., Yoshida M.M., Yacaman M.J.: The Completion of the Platonic Atomic Polyhedra: The Dodecahedron. Small. 3 (2), 351-355 (2006).

[5] Ermiş, T.: Düzgün Çokyüzlülerin Metrik Geometriler İle İlişkileri Üzerine. Ph.D. Eskişehir Osmangazi University (2014).

[6] Ermiş, T., Kaya, R.: Isometries the of 3-Dimensional Maximum Space. Konuralp Journal of Mathematics. 3 (1), 103-114 (2015).

[7] Gelişgen, Ö., Kaya, R.: The Taxicab Space Group. Acta Mathematica Hungarica. 122 (1-2), 187-200 (2009).

[8] Gelişgen, Ö., Çolak, Z.: A Family of Metrics for Some Polyhedra. Automation Computers Applied Mathematics Scienti c Journal. 24 (1), 3-15 (2015).

[9] Gelişgen, Ö., Can Z.: On The Family of Metrics for Some Platonic and Archimedean Polyhedra. Konuralp Journal of Mathematics. 4 (2), 2533 (2016).

[10] Gelişgen, Ö., Yavuz, S.: Isometry Groups of Chamfered Cube and Chamfered Octahedron Spaces. Mathematical Sciences and Applications E-Notes. 7 (2), 174-182 (2019).

[11] Griffiths, D.: Introduction to Elementary Particles. Wiley - VCH (1987).

[12] Horvath, A. G.: Semi-indefinite inner product and generalized Minkowski spaces. Journal of Geometry and Physics. 60 (9), 1190-1208 (2010).

[13] Horvath A. G.: Isometries of Minkowski geometries. Lin. Algebra and Its Appl. 512, 172-190 (2017).

[14] Lopez, J.L.R, Carrizales, J.M.M., Yacaman, M.J.: Low Dimensional Non-Crystallographic Metallic Nanostructures: Hrtem Simulation, Models and Experimental Results. Modern Physics Letters B. 20 (13), 725-751 (2006).

[15] Saller, H.: Operational Quantum Theory I-Nonrelativistic Structures.Springer-Verlag (2006).

[16] Schattschneider, D. J.: Taxicab group. Amer.Math. Monthly. 91, 423-428 (1984).

[17] Thompson, A.C.: Minkowski Geometry. Cambridge University Press, Cambridge (1996).

[18] http://dmccooey.com/polyhedra/RectifiedArchimedean.html

\section{Affiliations}

TEMEL ERMIŞ

AdDress: Eskişehir Osmangazi University, Science and Art Faculty, Deparment of Mathematics-Computer, 26480, Eskişehir, Turkey.

E-MAIL: termis@ogu.edu.tr

ORCID ID:0000-0003-4430-5271 